\title{
Extended gamma-ray emission of the solar flares in june 1991
}

\author{
G. Rank ${ }^{1}$, J. Ryan ${ }^{2}$, H. Debrunner ${ }^{3}$, M. McConnell ${ }^{2}$, and V. Schönfelder ${ }^{1}$ \\ 1 Max-Planck-Institut für Extraterrestrische Physik, PO Box 1312, 85741 Garching, Germany \\ 2 University of New Hampshire, Institute for the Study of Earth, Oceans and Space, Durham, NH 03824, USA \\ 3 Physikalisches Institut, Universität Bern, Sidlerstr. 5, 3012 Bern, Switzerland
}

Received 29 May 2001 / Accepted 19 July 2001

\begin{abstract}
During the solar flares on 9, 11, and 15 June 1991 the COMPTEL instrument measured extended $\gamma$-radiation in the $2.223 \mathrm{MeV}$ neutron-capture line, in prompt nuclear deexcitation lines and in pion-decay radiation for several hours after the flares. The long-term time profiles can be described by a double exponential decay with decay constants on the order of $10 \mathrm{~min}$ for the fast and several $100 \mathrm{~min}$ for the slow components. We studied the 11 June 1991 flare in more detail and found that during the extended phase the accelerated proton and ion spectrum is harder, the e/p ratio is lower, and the emission profile is smoother, compared to those of the impulsive phase. Pion-decay radiation was not detected before the onset of the extended emission phase. When comparing the three flares to one another, we found a striking similarity in the time profiles of the nuclear line and the neutron capture line emission. However, the pion-decay radiation varied in intensity significantly from flare to flare. The impulsive-phase emissions of the flares show no such similarity. Our measurements indicate that the processes taking place during the extended phase differ from those during the impulsive phase, or in other $\gamma$-ray line flares. Based on these results long-term trapping of energetic particles from the impulsive phase seems unlikely, as opposed to continuous particle acceleration.
\end{abstract}

Key words. Sun: activity - Sun: flares - Sun: X-rays, gamma rays

\section{Introduction}

During the two week period from 1 to 15 June 1991, active region NOAA 6659 moved across the solar disk and generated a series of major flares, including six X-class flares on $1,4,6,9,11$, and 15 June 1991 that were among the most powerful flares ever recorded. Several instruments observed the Sun during June 1991 and measured the high-energy emission from these events. The observations are summarized in Table 1. The active region was also monitored at optical wavelengths including magnetograms (e.g., Sakurai et al. 1992; Bumba et al. 1993).

For the Compton Gamma-Ray Observatory (CGRO) the Sun was declared a target-of-opportunity following the 4 June 1991 flare and the spacecraft was re-oriented to the solar direction on 9 June 1991. The flares on 9,11 and 15 June occurred within the field-of-view of all CGRO instruments. Here we present and discuss the $\gamma$-ray measurements of the three flares obtained with COMPTEL.

Send offprint requests to: G. Rank,

e-mail: gzr@mpe.mpg.de
The X10/3B flare on 9 June 1991 started at 0136 UT, according to the GOES-7 SXR monitor. It was located at N34E04 (Solar Geophysical Data, 1991). Gamma-ray observations by OSSE were reported by Murphy et al. (1993). EGRET/TASC measurements show high energy emission well after the impulsive phase (Schneid et al. 1994). COMPTEL measured both $\gamma$-rays and neutrons (Ryan et al. 1994), indicating that the bulk of the neutrons was emitted after the peak in the $\gamma$-radiation, and prolonged emission in the $2.223 \mathrm{MeV}$ neutron capture line, the most prominent spectral feature in the COMPTEL energy range, was reported by Rank et al. (1997).

The X12/3B flare on 11 June 1991 started at 0156 UT at an $\mathrm{H} \alpha$ location of N31W17 (Solar Geophysical Data, 1991). The EGRET spark chamber registered $>50 \mathrm{MeV}$ $\gamma$-radiation for a period of 11 hours (Kanbach et al. 1993). Measurements of the impulsive phase with the EGRET/TASC were reported by Schneid et al. (1994) and Dunphy et al. (1999). COMPTEL registered a signal in the neutron capture line for several hours (Ryan et al. 1993a; Rank et al. 1993; Rank 1996), demonstrating that a fraction of the prolonged radiation originated 
Table 1. High-energy observations of three X-class flares in June 1991.

\begin{tabular}{lllll}
\hline Instrument & Energy Range & 9 June 1991 & 11 June 1991 & 15 June 1991 \\
\hline COMPTEL & 0.8 to $30 \mathrm{MeV}$ & several hours & several hours & $\begin{array}{l}\text { several hours, } \\
\text { w/o imp. phase }\end{array}$ \\
& $\begin{array}{l}10-100 \mathrm{MeV} \\
\text { neutrons }\end{array}$ & about 1 hour & about 1 hour & several hours \\
BATSE-LAD & $20 \mathrm{keV}$ to $>1 \mathrm{MeV}$ & about 1 hour & about 1 hour & about 40 min \\
OSSE & $40 \mathrm{keV}$ to $>150 \mathrm{MeV}$ & imp. phase & about 2 hours & - \\
EGRET & $>30 \mathrm{MeV}$ & - & several hours & - \\
GAMMA-1 & $>50 \mathrm{MeV}$ & (TASC: imp. phase) & (TASC: imp. phase) & several hours \\
PHEBUS & $75 \mathrm{keV}$ to $>1 \mathrm{MeV}$ & imp. phase & - & - \\
\hline
\end{tabular}

from energetic ions. Prolonged emission in the $2.223 \mathrm{MeV}$ line was also reported by Murphy et al. (1993) using OSSE data. COMPTEL also directly measured neutrons form this flare over a prolonged period (Ryan et al. 1993a; Rank et al. 1994; Rank 1996). Finally, observations were performed by PHEBUS/GRANAT measuring bremsstrahlung, the $2.223 \mathrm{MeV}$ line, and nuclear emission during the impulsive phase (Trottet et al. 1993; Trottet 1994).

The solar flare on 15 June 1991 was a 3B/X12 event and started at $0810 \mathrm{UT}$. By this time active region NOAA 6659 had rotated to N33W69 (Solar Geophysical Data, 1991). The impulsive phase was not observed with any high-energy instrument. GAMMA-1 measured a declining signal of $>50 \mathrm{MeV}$ radiation during a window starting about $30 \mathrm{~min}$ after flare onset, and registered a detectable signal in the next spacecraft orbit (Akimov et al. 1991, 1994; Kocharov et al. 1994). With COMPTEL McConnell et al. (1993) measured $\gamma$-ray emission during a time window that started about $40 \mathrm{~min}$ after flare onset, and also for the following spacecraft orbits (Rank et al. 1996). Flare neutrons were measured with COMPTEL for two orbits (Debrunner et al. 1993; Rank 1996; Nieminen 1997). A combined analysis of COMPTEL and GAMMA-1 data during the extended phase was presented by Kocharov et al. (1998).

The most remarkable result of the June 1991 flares was the observation of prolonged emission in $\gamma$-rays, hard X-rays and neutrons, for as long as 11 hours after the flare onsets. This implies that accelerated protons and ions of high energies were present at the flare site for a substantial fraction of a day. The intensity-time profiles for the $2.223 \mathrm{MeV}$ line are remarkably similar for all three flares (Rank et al. 1996). Different explanations for the origin of the $\gamma$-ray emission during the extended flare phases were proposed:

A model for the 11 June 1991 flare (Mandzhavidze \& Ramaty 1992; Ramaty \& Mandzhavidze 1994) based on the $>50 \mathrm{MeV}$ measurements by EGRET explained the extended emission by protons and ions that were accelerated during the impulsive phase and subsequently trapped in non-turbulent coronal magnetic loops. It was pointed out by Akimov et al. (1991) using the microwave lightcurve of the 15 June 1991 flare and the $\gamma$-ray time profiles measured by GAMMA-1 that both types of emission behaved similarly despite originating from different parent particle populations. Rank et al. (1996) reported a similar behaviour for different components of the $\gamma$-ray spectrum that originate from protons of different energies. They studied the $2.223 \mathrm{MeV}$ line, 4-7 MeV, and 8-30 MeV time profiles for the first hour of the 11 June 1991 flare as measured by COMPTEL, as well as the long-term $2.223 \mathrm{MeV}$ line profile from COMPTEL and the $>50 \mathrm{MeV}$ profile from EGRET.

Here we summarize the COMPTEL measurements of the extended $\gamma$-ray emission for the flares on 9,11 , and 15 June 1991 including the long-term time profiles in the 4-7 MeV and 8-30 MeV band. Compared to earlier publications we used an improved CGRO mass model for simulating the instrument response. We also include BATSE observations for an independent measure of the electron bremsstrahlung spectrum.

In Sect. 2 we describe the basic principle of COMPTEL with emphasis on solar observations, and the analysis techniques we used. In Sect. 3 we present the data. In Sect. 4 we discuss our measurements, compare to the results of other instruments, and relate our findings to models of particle acceleration and transport. We summarize our conclusions in Sect. 5.

\section{Instrumental description}

\subsection{The COMPTEL telescope}

COMPTEL is one of four instruments on board the Compton Gamma-Ray Observatory that was launched in 
April 1991. A full description of COMPTEL was presented by Schönfelder et al. (1993).

The COMPTEL instrument was designed for imaging and spectroscopy of $\gamma$-rays in the range from 0.75 to $30 \mathrm{MeV}$. It consisted of two layers of detector modules separated by $1.58 \mathrm{~m}$. The upper (D1) detector layer consisted of seven liquid scintillator detector modules, optimized for a single Compton scattering of a $\gamma$-ray photon in the MeV range. Each D1 module was $23 \mathrm{~cm}$ in diameter with a height of $8.5 \mathrm{~cm}$, being filled with liquid organic scintillator NE213A. The lower (D2) detector layer consisted of $14 \mathrm{NaI}$ scintillator modules, thick enough to absorb the scattered photon. Each D2 module had a diameter of $28 \mathrm{~cm}$ and a height of $7.5 \mathrm{~cm}$. A number of photomultiplier tubes (PMT) were attached to each detector module to measure the energy deposit. From the relative pulse heights of the PMTs the location of the interaction in the modules was calculated while the sum of the PMT signals provided the deposited energy.

Each of the detector layers was enclosed by $1.5 \mathrm{~cm}$ thick anticoincidence shields. The shields had a detection efficiency for charged particles better than 99.9\%. They also attenuated soft X-ray photons which would contribute to pulse pile-up effects. At higher energies, the attenuation decreases from about $95 \%$ at $10 \mathrm{keV}$ to $5 \%$ at $1 \mathrm{MeV}$.

COMPTEL had two modes of data acquisition that operated in parallel: the telescope or double scatter mode, and the single detector mode.

The COMPTEL telescope mode utilized a doublescatter technique. In an ideal event, an incoming $\gamma$-ray photon Compton-scatters once within one of the D1 modules. The scattered photon is then fully absorbed in one of the D2 modules. From the measured energy deposits $E_{1}$ and $E_{2}$ in the D1 and D2 detector, the energy of the incoming $\gamma$-ray photon is

$E_{\gamma}=E_{1}+E_{2}$.

Since the energy of the scattered photon before and after the Compton interaction (denoted as $E_{\gamma}$ and $E_{2}$ ) is known, the scatter angle $\bar{\varphi}$ used in the imaging process is

$\cos \bar{\varphi}=1-\frac{m_{\mathrm{e}} c^{2}}{E_{2}}+\frac{m_{\mathrm{e}} c^{2}}{E_{1}+E_{2}}$.

In solar flare studies, the imaging enables one to suppress background. For each event, parameters such as the time-of-flight (TOF) from the D1 to the D2 detector or a pulse shape discrimination (PSD) in the D1 signal were recorded. The combination of the double scatter technique, the anticoincidence shields and additional event selection criteria enabled measurements with high sensitivity. However, the system was prone to dead time problems when the fluxes were high, e.g., during the impulsive phase of large flares.

In its double-scatter mode COMPTEL was also sensitive to neutrons in the range of about 10 to $100 \mathrm{MeV}$. By replacing the Compton interaction (Eq. (2)) with the equivalent kinematic expression for non-relativistic neutrons, COMPTEL could measure the source direction and energy of a neutron.

Two of the D2 modules were operated independently as spectrometers. They were called the high-range and low-range burst modules (HRBM and LRBM). During June 1991 only the high-range burst detector was active, collecting data in the range from about 0.6 to $11 \mathrm{MeV}$. The on-board processing and telemetry for these modules was performed separately and suffered only minor dead time effects. The sensitivity of the burst modules was significantly poorer than of the telescope mode. However, for large flares, the telescope and burst modes complemented one another: the burst mode provided a virtually deadtime free count rate during the impulsive phase, while the better sensitivity of the telescope mode permitted the study of low intensity emission.

\subsection{Data analysis}

COMPTELs angular resolution of $>1^{\circ}$ does not allow one to resolve the solar disk. However, since the source position is known, imaging was used to suppress background in the following way: not all events are fully absorbed in the lower detector and hence the determined scatter angle $\bar{\varphi}$ does not always equal the true geometrical scatter angle $\varphi_{\text {geo }}$. The deviation $\left|\bar{\varphi}-\varphi_{\text {geo }}\right|$ is called angular resolution measurement (ARM). For a known source position, events with a large ARM have either not been fully absorbed or they are not consistent with solar origin and are likely background events. Further, $\bar{\varphi}$ is linked to the energy of the photon through Eq. (2). By rejecting events with a large ARM it is assured that the photons are consistent with a solar origin and that no systematic effects influence the energy determination. For our analysis, we have set the upper ARM limit to accept an event to $3 \sigma_{\mathrm{A}}$, where $\sigma_{\mathrm{A}}$ is the angular resolution of COMPTEL.

Most of the instrumental background originates from cosmic rays. The intensity of the cosmic radiation varies with the local rigidity of the Earth's magnetic field and, therefore, with the position of the spacecraft. Thus, the background level for the spacecraft at a given time can be estimated from intervals before and after the flare when the relative positions of the Earth, Sun and the spacecraft are similar. During June 1991 the orbital configuration could be reproduced accurately enough with a periodicity of either 15 or 16 CGRO orbits (about 1 day).

For the flare on 11 June 1991 we used four background intervals, located 15 and 16 orbits before and after the flare. We averaged the counts from all four intervals. In the case of the 9 June flare we only used the two intervals located 15 and 16 orbits after the flare, since CGRO changed its pointing shortly before the flare started. Also, after the 15 June flare the instrument was re-oriented so that only two intervals located 15 and 16 orbits before the flare could be used. 


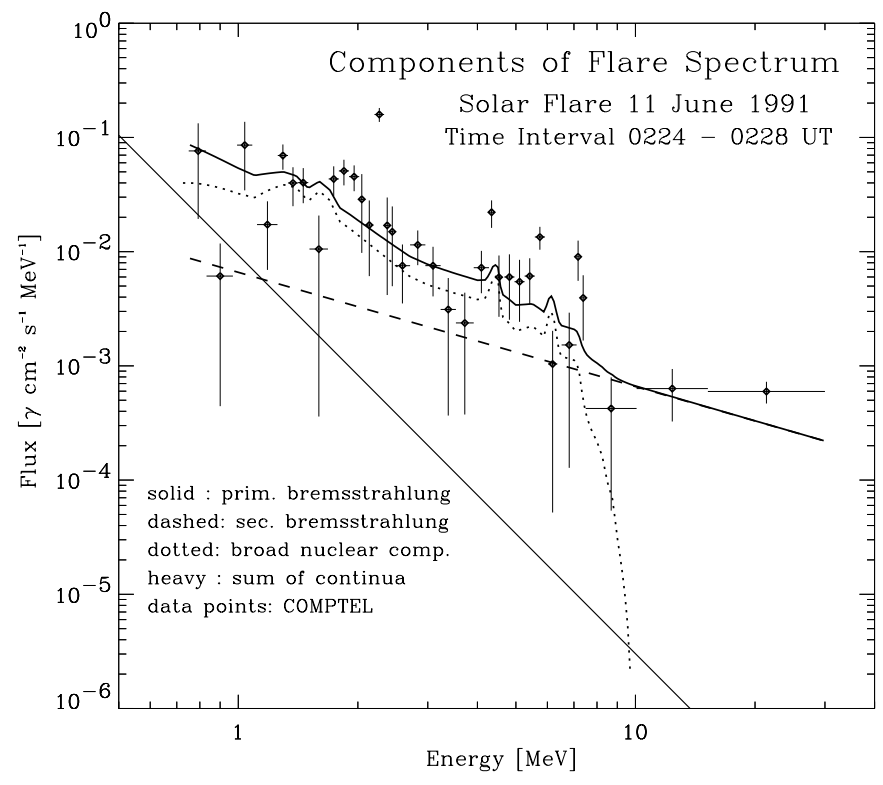

Fig. 1. Separation of spectral components for a time interval during the extended phase of the 11 June flare (see text).

The validity of the background subtraction was ensured by analyzing two time intervals at the end of the day on 9 and 11 June where no GOES or BATSE events have been reported. Both results have led to a signal consistent with zero.

During the impulsive phase of a large solar flare the intense soft X-ray flux saturated the anti-coincidence shields and led to severe instrumental dead time effects. During the June 1991 observation period the telemetry rate was limited to 24 events per second. Both the telemetry limit and the high trigger rate of the anti-coincidence detectors produced significant dead time effects. The validity of the dead time correction procedure (van Dijk 1996) was tested by comparing the time profiles of corrected telescope fluxes with the count rates of the HRBM over the same energy intervals.

\subsection{Spectral and temporal analysis}

To generate flux spectra for a given time interval, count rate spectra are integrated, corrected for detector deadtime and background, and then deconvolved using a Maximum Entropy Method. The detector response was simulated with a Monte-Carlo calculation verified by calibration measurements (Stacy et al. 1996). Since the instrumental response depends on the position of the source in the field-of-view, a separate response matrix was generated for each flare.

The solar observation intervals are constrained by the CGRO orbit, the occultation of the Sun by the Earth, and by passages through the South Atlantic Anomaly (SAA) during which the instrument's high voltage is switched off. Under favourable geometric conditions an observation can be as long as one hour. To obtain time profiles of the $\gamma-$ ray flux the observation intervals were subdivided. Each of these periods is long enough to provide a good statistics for spectral deconvolution.

To determine the bremsstrahlung spectrum below the nuclear line range $(<0.45 \mathrm{MeV})$ we used data from BATSE and PHEBUS. To isolate the nuclear emission we extrapolated the bremsstrahlung continuum to higher energies and subtracted it from the deconvolved COMPTEL telescope spectrum. When we could not deduce the bremsstrahlung spectrum, we used a single power law with a fixed index and jointly fitted this power law with the other spectral components. Late in the extended flare phases the bremsstrahlung signal was too weak to measure, so we used the spectral shape from earlier in the flare.

During the impulsive phases of the 11 June 1991 flare we used measurements of PHEBUS to determine the index. For the the impulsive phases of the 9 June 1991 flare there are no other measurements and we used the index derived during the extended phase.

During the extended phase of the 11 and 15 June 1991 flares the signal in the energy range 8-30 MeV exceeds the extrapolated bremsstrahlung continuum. We ascribe the excess to pion decay radiation, including the emission from secondary bremsstrahlung. The spectral form of the secondary bremsstrahlung was studied by Murphy et al. (1987). In the energy range below $30 \mathrm{MeV}$ it can well be approximated by a power law function $\propto E^{-1}$. We used this simple template and fitted it to the data above the nuclear line region. The contribution of the secondary bremsstrahlung to the 4-7 MeV range exceeds that of the primary bremsstrahlung but is below $20 \%$ of the total in all cases. For the $2.223 \mathrm{MeV}$ line the influence of the secondary bremsstrahlung component is not significant.

To obtain the nuclear line flux we integrated our measured flux over the energy band 4-7 MeV and subtracted the contribution from primary and secondary bremsstrahlung. For the $2.223 \mathrm{MeV}$ line, the contribution of nuclear lines was also subtracted. We fitted the broad nuclear line component with a template that we normalized to the measured spectrum in the energy range 3-4 MeV where no strong narrow nuclear lines are present. The template was taken from the broad nuclear component of the 21 April 1981 flare (Murphy et al. 1990). We assumed that the form of the nuclear continuum does not significantly differ between the two flares. With this assumption the $2.223 \mathrm{MeV}$ line flux is assigned to the remaining flux within $\pm 1 \sigma$ of the $2.223 \mathrm{MeV}$ line position. Our results are consistent with those of Rank et al. (1996), however, the template fitting used here is more robust, particularly for spectra with low count rates.

In Fig. 1 we show an example of separated spectral components. The spectrum taken from a 4 min interval during the extended phase of the 11 June 1991 flare includes a strong contribution from pion decay radiation. The data points represent the measured $\gamma$-ray flux spectrum of COMPTEL. Three continuum components are indicated in the figure: a power law $\propto E^{-3.3}$ representing the primary bremsstrahlung continuum (thin solid line), the 


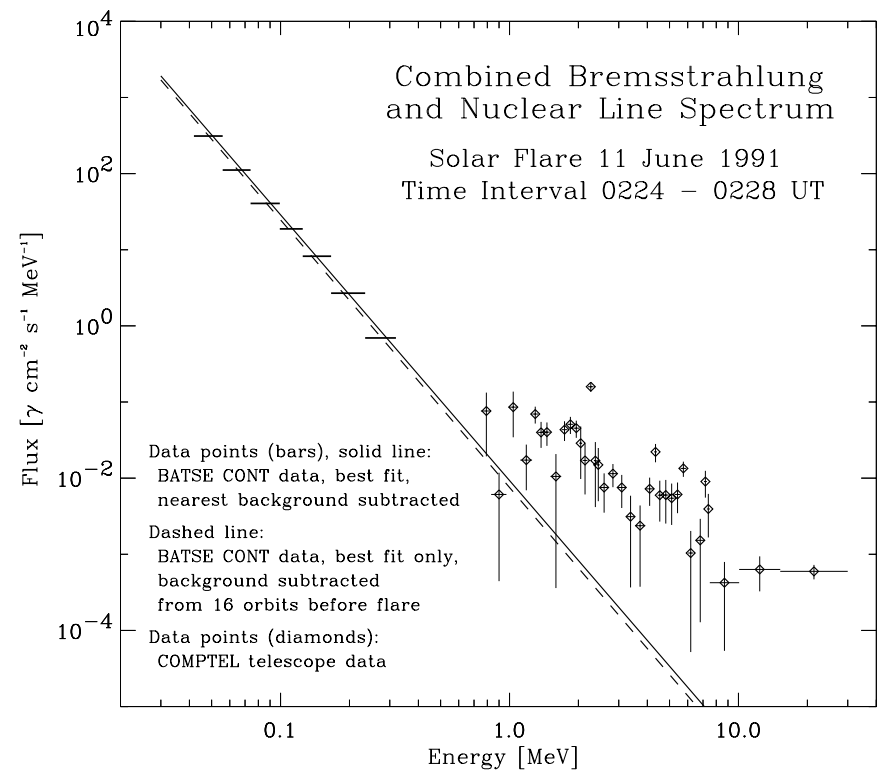

Fig. 2. Combined spectrum for the 11 June flare. Results from different evaluation methods for the bremsstrahlung are shown.

template for the nuclear continuum (dotted line), and the power law $\propto E^{-1}$ as an approximation for the secondary bremsstrahlung component. The sum of all three continua is indicated (heavy solid line).

\section{Observational results}

\subsection{Bremsstrahlung spectrum from BATSE and PHEBUS data}

In order to separate the nucleonic and electronic components in the COMPTEL spectra we must determine the contribution of the primary electron bremsstrahlung to the signal. We analyzed hard X-ray data from the instruments BATSE/CGRO and PHEBUS/GRANAT for independent measures of the bremsstrahlung continuum.

The BATSE large area detectors (LAD) measured hard X-rays in the energy range from about $20 \mathrm{keV}$ to a few MeV. During the impulsive phase of the flares on 9 and 11 June the LAD detectors suffered dead time effects so we did not use these data. However, during the extended phase of both flares the fluxes decreased sufficiently so that the signals can be analyzed. For the 15 June flare the impulsive phase was not observed and when BATSE resumed the observations, dead time effects were not a problem.

Data from the BATSE LADs are provided in two formats: DISCLA data have a time resolution of $1.024 \mathrm{~s}$ in four energy channels; CONT data provide a higher energy resolution with 16 energy channels between about $20 \mathrm{keV}$ and $>2 \mathrm{MeV}$ with a time resolution of $2.048 \mathrm{~s}$. We used CONT data to study the bremsstrahlung spectrum. Data above $427 \mathrm{keV}$ (channel 11 and higher), where nuclear line emission begins to contribute to the signal, were excluded from the spectral fitting. Only data from the most sunward facing detector were used.
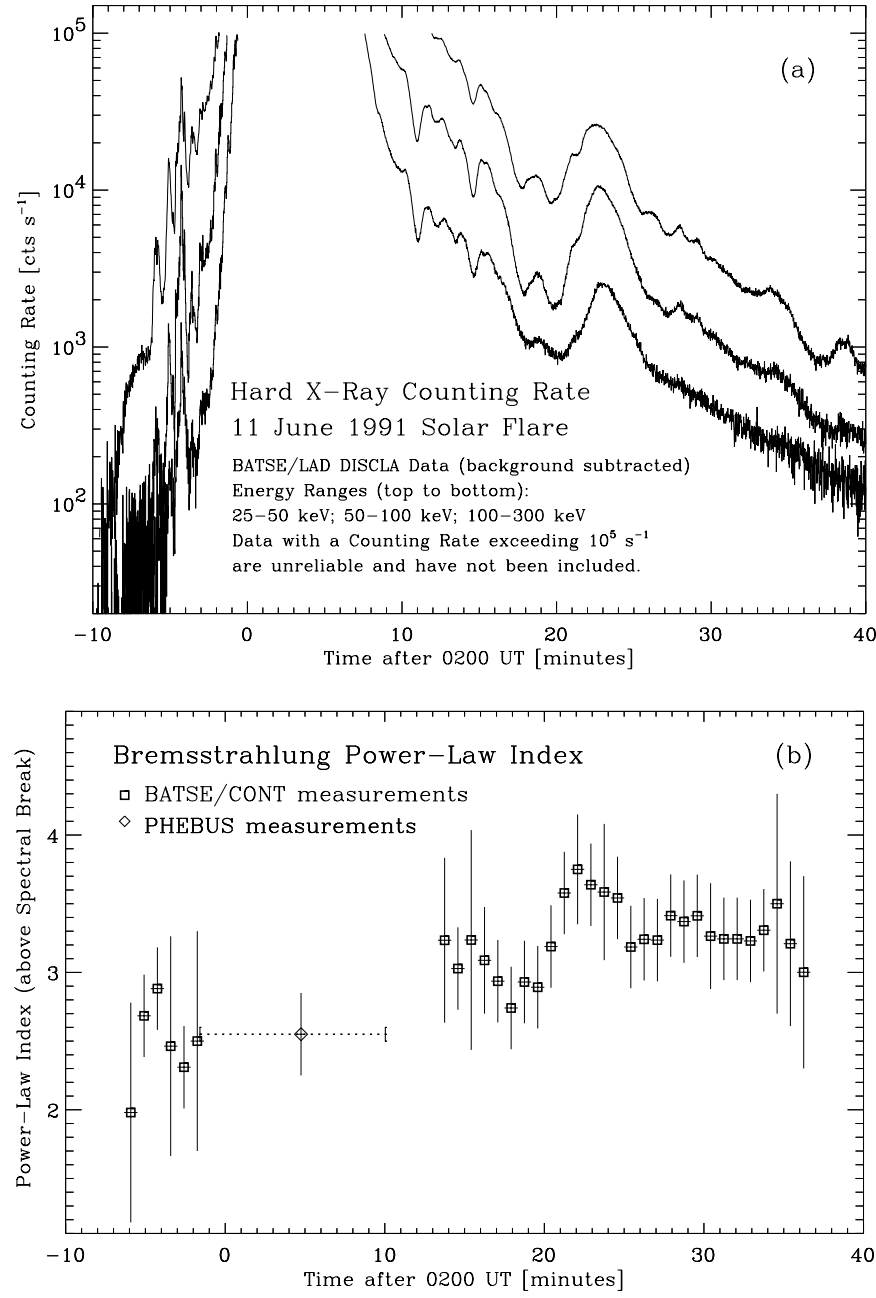

Fig. 3. a) DISCLA counting rate of the most sunward-facing BATSE-LAD for three energy channels. The data during the impulsive phase are unreliable and have been omitted. b) The power-law indices deduced from CONT data by the fitting procedure for $50 \mathrm{~s}$ time intervals. If a broken power-law was fitted, only the index above the spectral break is shown. The error bars represent the sum of statistical and systematic errors. Additionally, the bremsstrahlung index measured by PHEBUS/GRANAT during flare maximum is included. The whole observation period of PHEBUS is indicated by the dotted range.

For analyzing the CONT data we used the package SPEX which is part of the solar software library. The data were background subtracted and corrected for instrumental live time effects. To perform the deconvolution, a model spectrum was selected. Based on a forward-folding of the model spectrum, the free parameters were optimized by minimizing $\chi^{2}$, yielding a conversion factor between the measured counts and the photons. The full detector response was necessary for the zenith angle of the Sun for that LAD detector.

For the 9 and 11 June flare, we avoided times near the impulsive phases. The detector dead time for all analyzed intervals was below $50 \%$. This excludes all times

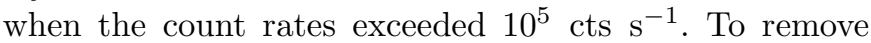


the background we used background models based on the count rate measured directly before and after the flare. For the long-duration events in June 1991 only a background interval before flare onset could be used. However, the result is not very sensitive to the defined background interval, since the flare count rate greatly exceeded the background count rate.

For the 11 June 1991 flare a more detailed analysis was performed to estimate the systematic effects of the background subtraction. We tested a model background derived from a time interval 16 orbits before the 11 June 1991 flare where the orbital conditions were similar to those of the flare (this concept is also used for the COMPTEL analysis, Sect. 2.2). For this comparison, we excluded data below $45 \mathrm{keV}$ to avoid being affected by a superhot thermal component.

The resulting bremsstrahlung spectra for both background models are plotted in Fig. 2, together with the deconvolved COMPTEL telescope spectrum. Data are taken for the same time interval as used in Fig. 1. The results of both background subtraction techniques agrees well.

To study the development of the bremsstrahlung spectrum of the 11 June 1991 flare in greater detail we fitted spectra for time intervals of $50 \mathrm{~s}$ at the beginning of the impulsive phase and during the extended phase. Figure $3 \mathrm{a}$ shows the time profile using the count rates in the three lower DISCLA channels from $25 \mathrm{keV}$ to $300 \mathrm{keV}$.

For each $50 \mathrm{~s}$ interval we fitted both a broken and a single power law to the CONT data. Except for a few cases, the $\chi^{2}$ value alone was sufficient to decide between the models. The resulting power law indices are displayed in Fig. 3b. For spectra with good statistics the fitting errors are less than a few percent. By varying the background intervals and the energy range of the fit we estimated the systematic error to be between $5 \%$ and $10 \%$. The errors indicated in Fig. 3b are the geometric sum of statistical and systematic errors.

In general, we found that prior to the flare maximum a broken power law is the best fit. Typical power-law indices of about 5 or 6 below the break, and about 2.5 above the break fit the data. The break appears at energies of about $80 \mathrm{keV}$. In Fig. 3b only the index above the spectral break is shown. Fitting an additional superhot component yielded large and erratic plasma temperatures on the order of several hundred MK and large $\chi^{2}$ values.

After the flare maximum a superhot component is necessary to describe the spectra. Starting around 0210 UT when the live time rose above $50 \%$, the measured plasma temperature deduced for the superhot component is about $20 \mathrm{MK}$ increasing smoothly to a value of about 50 to $60 \mathrm{MK}$ around $0230 \mathrm{UT}$. The deduced plasma temperature is in the same range as the highest temperature reported by Lin et al. (1987) from their study of 35 smaller flares. The power-law index appears to be larger after the flare maximum. It shows a significant rise around $0214 \mathrm{UT}$, coincident in time with a burst seen in all HXR channels. The plasma temperature of the superhot component does not change significantly during the burst.
An independent measurement was done with the PHEBUS instrument on GRANAT during the impulsive phase of the 11 June flare down to $75 \mathrm{keV}$ (Trottet et al. 1993). The bremsstrahlung continuum as measured with PHEBUS for the 11 June flare can by described by a power law index of $3.5 \pm 0.4$ for the count rate spectrum (Trottet et al. 1993; Trottet 1994), and $2.55 \pm 0.4$ for the flux spectrum (Trottet, private communication). Furthermore, no significant change in the spectral index was detected throughout the observation period of PHEBUS (Barat, private communication).

The spectra derived from PHEBUS and BATSE agree. The value of 2.55 obtained by PHEBUS agrees with the value during the impulsive phase as determined with BATSE. No spectral break was observed by PHEBUS. However, the break observed by BATSE appeared at about the threshold energy of PHEBUS. The PHEBUS data are included in Fig. 3b. The horizontal range indicates that the spectral index did not change during this time interval.

\subsection{The solar flare on 11 June 1991}

The flare on 11 June 1991 started at 0156 UT according to GOES-7 measurements. The orbital daytime period for CGRO started at about $0150 \mathrm{UT}$, and the CGRO instruments observed the flare emission without interruption until orbital night at about 0300 UT. The BATSE solar flare trigger occurred at 0153:37 UT, but a signal in the HXR range of $25-50 \mathrm{keV}$ was already present at about 0151 UT (Fig. 3). The first significant emission in the COMPTEL energy range was detected in the telescope mode at $0158 \mathrm{UT}$.

Due to the intense flux of X-rays and $\gamma$-rays the sensitivity of COMPTEL was degraded by dead time effects during the impulsive phase. The main reason for the dead time was a high trigger rate of the anti-coincidence detector system, caused by pulse pile-up of SXR photons (see Sect. 2.2). However, due to the comparatively slow development of the impulsive phase and the possibility to analyze the virtually dead time free HRBM data it is possible to study the emission during the impulsive phase in some detail.

Following the technique described in Sect. 2.3 we measured the fluxes in the $2.223 \mathrm{MeV}$ neutron capture line, and the energy bands $4-7 \mathrm{MeV}$ and $8-30 \mathrm{MeV}$ for 12 time intervals between 0158 UT and 0300 UT. These are displayed in Fig. 4a. The HRBM data with a time resolution of $12 \mathrm{~s}$ are also shown (arbitrarily scaled). During the impulsive phase the prompt nuclear line emission is pronounced with increasing intensity in the $2.223 \mathrm{MeV}$ line. Only upper limits are derived for the bremsstrahlungcorrected 8-30 MeV channel. After the flare maximum the nuclear line flux falls to a low level for several min. The $2.223 \mathrm{MeV}$ line, however, remains prominent, slowly declining until 0300 UT. After about 0213 UT the nuclear line flux quickly rises to a maximum at about $0215 \mathrm{UT}$ 

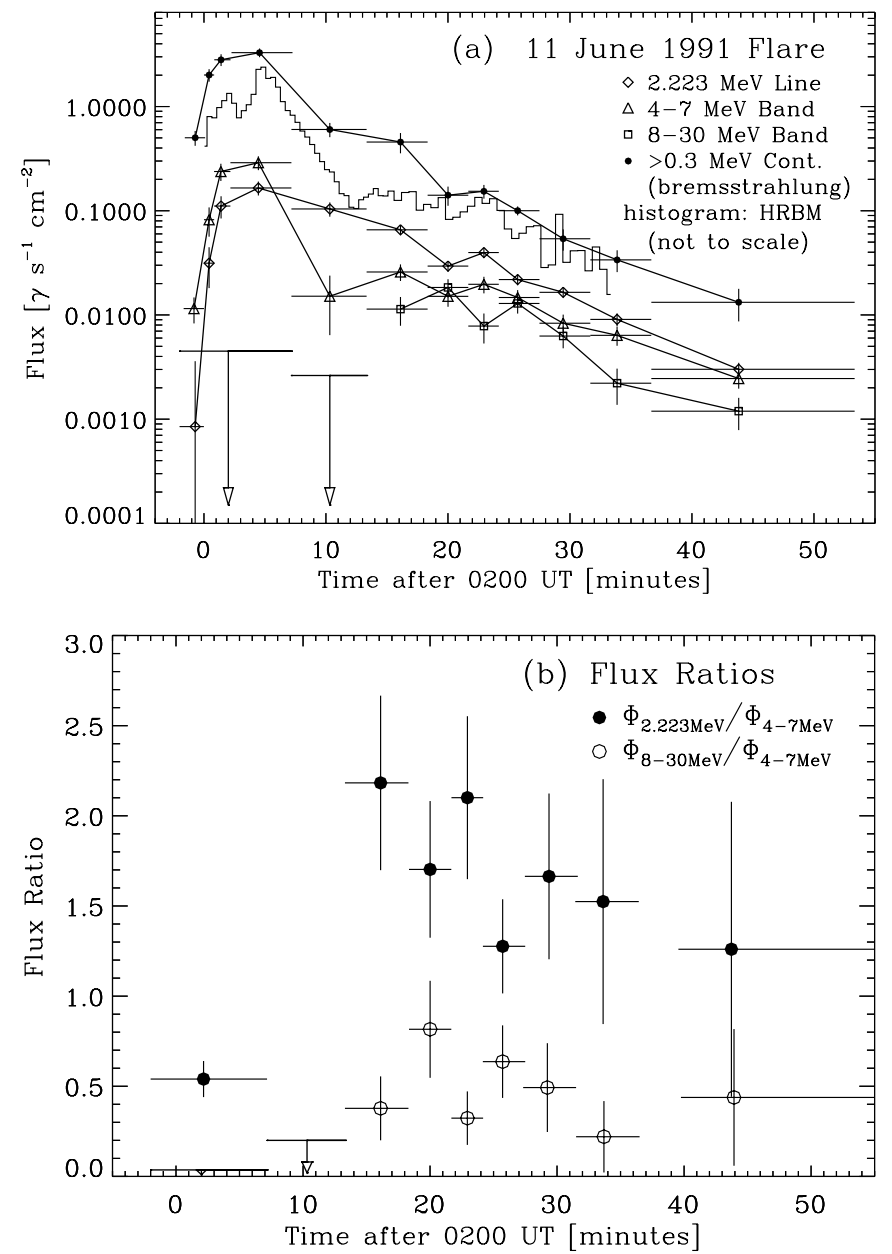

Fig. 4. Time profile for fluxes a) and flux ratios $\mathbf{b}$ ) of different spectral components during the first hour of the 11 June 1991 flare. Vertical bars are $1 \sigma$ errors, and horizontal bars indicate the duration of the analyzed time intervals. For the $8-30 \mathrm{MeV}$ radiation two $1 \sigma$ upper limits are given. The arbitrarily scaled HRBM counting rate has a time resolution of $12 \mathrm{~s}$.

and slowly decays thereafter. A clear signal appears in the $8-30 \mathrm{MeV}$ channel after 0213 UT. We emphasize that the electron bremsstrahlung is subtracted from the data shown in Fig. 4a. Therefore, we attribute the emission in the 8-30 MeV range to pion decay.

The bremsstrahlung correction after 0213 UT was done using BATSE/CONT spectra as explained in Sect. 3.1. For the impulsive phase we fitted a power law with a fixed index of 2.55 as measured with PHEBUS. The integrated bremsstrahlung flux above $300 \mathrm{keV}$ is also included in Fig. 4a. The bremsstrahlung varies in a similar way to the nucleonic components. After 0213 UT it falls off in a similar manner as the other components.

We fitted exponential curves to the decaying fluxes after 0213 UT. We obtained fits with $\chi^{2}$ values between 1.5 and 3.0, and values for the time constant of $9.5 \pm 0.5 \mathrm{~min}$ for the $2.223 \mathrm{MeV}$ line, $12.1 \pm 1.1 \mathrm{~min}$ for the $4-7 \mathrm{MeV}$ band, $10.8 \pm 1.4 \mathrm{~min}$ for the $8-30 \mathrm{MeV}$ band, and $8.6 \pm 1.0 \mathrm{~min}$ for the $>300 \mathrm{keV}$ bremsstrahlung emission.

We use the ratio between the $2.223 \mathrm{MeV}$ line and the 4-7 MeV flux as an indicator of the spectral hardness of the proton spectrum in the energy range of about 10-100 MeV. Because the neutron capture line radiation is emitted over a time of 1 or $2 \mathrm{~min}$ the fluxes cannot be compared directly. Either the fluences must be compared for time intervals that are much longer than the neutron capture time of about $70 \mathrm{~s}$, or the production and decay of the radiation must be modelled. We used both approaches and compare the results.

For the impulsive phase, time intervals that are sufficiently long to deconvolve the spectra are on the order of the expected neutron capture time. Therefore, we integrated the flux over the whole impulsive phase (0158 to $0207 \mathrm{UT}$ ). The resulting time interval of $550 \mathrm{~s}$ is much longer than the neutron capture time. We found fluences of $\Phi_{2.2}=60.4 \pm 7.8 \gamma \mathrm{cm}^{-2}$ for the $2.223 \mathrm{MeV}$ line and $\Phi_{4-7}=74.4 \pm 10.8 \gamma \mathrm{cm}^{-2}$ for the $4-7 \mathrm{MeV}$ range. These yield a fluence ratio for the impulsive phase of $\Phi_{2.2} / \Phi_{4-7}=0.82 \pm 0.15$. Compared to the results of Rank (1996) the fluence values are lower by more than a factor of 2 . The ratio, however, is unchanged over the entire event. The lower fluences reflect changes in the response matrices from improvements in the CGRO mass model used in the response simulations.

All time intervals after $0207 \mathrm{UT}$ are much longer than $70 \mathrm{~s}$. The $\Phi_{2.2} / \Phi_{4-7}$ ratios are shown in Fig. 4b. The first time interval following the impulsive phase (from 0207 to 0213 UT) has a high value of $\Phi_{2.2} / \Phi_{4-7}=5.6 \pm 2.5$ (data point not included in the figure). After $0213 \mathrm{UT}$ the ratio is between 1 and 2 with a trend to decrease until 0300 UT.

We also employed the technique first used by Prince et al. (1983) to model the time profile of the neutron capture line. The measured flux of the prompt nuclear line emission in the $4-7 \mathrm{MeV}$ range is taken as a template for the neutron-production time profile. The model parameters are the capture time constant $\lambda$ and the spectral hardness ratio $R$ that takes into account neutrons are generated at higher energies than the prompt nuclear lines. We computed the expected $2.223 \mathrm{MeV}$ line profile and adjust it to the measured profile.

The telescope data are not well suited for this analysis because of the large dead time effects during the impulsive phase. Instead, we use HRBM data with a time resolution of $12 \mathrm{~s}$. The HRBM data are not deconvolved and we only have relative values for the spectral hardness $\left(R^{\prime}\right)$. The 2.0-2.4 MeV interval that also contains continuum components is a proxy for the $2.223 \mathrm{MeV}$ line, but from the deconvolved telescope spectra we know that the line dominates this interval (Sect. 3.1).

The results of the model fits are displayed in Fig. 5. The measured count rates in the energy bands 2.0-2.4 MeV and 4.0-7.0 MeV are shown as histograms. The prompt nuclear emission falls below the sensitivity limit of the HRBM detector after the impulsive phase while the $2.223 \mathrm{MeV}$ emission decays relatively slowly. 


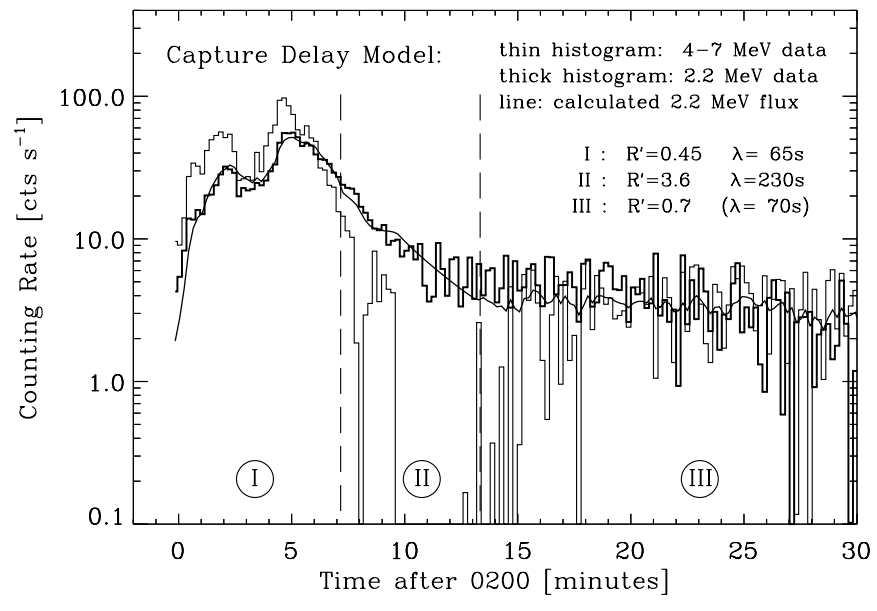

Fig. 5. Model for the $2.223 \mathrm{MeV}$ time profile. The best fit parameters for the three ranges are displayed.

This is consistent with the fact that the 4-7 MeV signal in the telescope data almost vanishes after the impulsive phase (Fig. 4a).

We were unable to describe the whole time interval with one set of parameters. Instead, the flare had to be divided into three parts as indicated in Fig. 5. During the impulsive phase (denoted as part I in the figure) the emission is well structured and yields a $\lambda$ of $(65 \pm 10) \mathrm{s}$. The hardness parameter is $R^{\prime}=0.45 \pm 0.1$. After the impulsive phase (part II) we determined $R^{\prime}=3.6 \pm 1.2$ and $\lambda=230 \mathrm{~s}$ with a $1 \sigma$ range of $140-390 \mathrm{~s}$. At later times (part III) there is no structure in the time profile that allows us to determine $\lambda$. Therefore, we used a fixed value of $\lambda=70 \mathrm{~s}$ and determined $R^{\prime}=0.7 \pm 0.3$. The result is not very sensitive to our choice of $\lambda$. As with the estimate of $\Phi_{2.2} / \Phi_{4-7}$ the fitting of the neutron capture time shows variations in the spectral hardness ratio $R^{\prime}$ that accompanies a significantly changing neutron capture time.

In Fig. $4 \mathrm{~b}$ we also include the ratio $\Phi_{8-30} / \Phi_{4-7}$. Since the pion-decay emission in the $8-30 \mathrm{MeV}$ range probes the proton spectrum at several hundred $\mathrm{MeV}$, while the 4-7 MeV photons originate from $10-20 \mathrm{MeV}$ protons, the ratio is sensitive to the hardness of the accelerated proton spectrum at energies around $100 \mathrm{MeV}$. However, since the range $8-30 \mathrm{MeV}$ contains only a small fraction of the pion decay emission, the ratio does not easily permit us to obtain absolute hardness parameters. The emission in both components is radiated promptly and can be compared directly. Before the extended phase only upper limits are obtained. Combining the data during the extended phase we obtain a ratio of $0.62 \pm 0.08$.

We determined the ratio between the $>300 \mathrm{keV}$ bremsstrahlung flux and the 4-7 MeV nuclear line emission, $\Phi_{\text {brems }} / \Phi_{4-7}$, both of which are emitted promptly. Since the bremsstrahlung continuum is emitted by electrons and the nuclear lines originate from reactions caused by accelerated protons, $\Phi_{\text {brems }} / \Phi_{4-7}$ is sensitive to the electron-to-proton ratio in the flare. We integrated the $>300 \mathrm{keV}$ bremsstrahlung flux during the three phases as
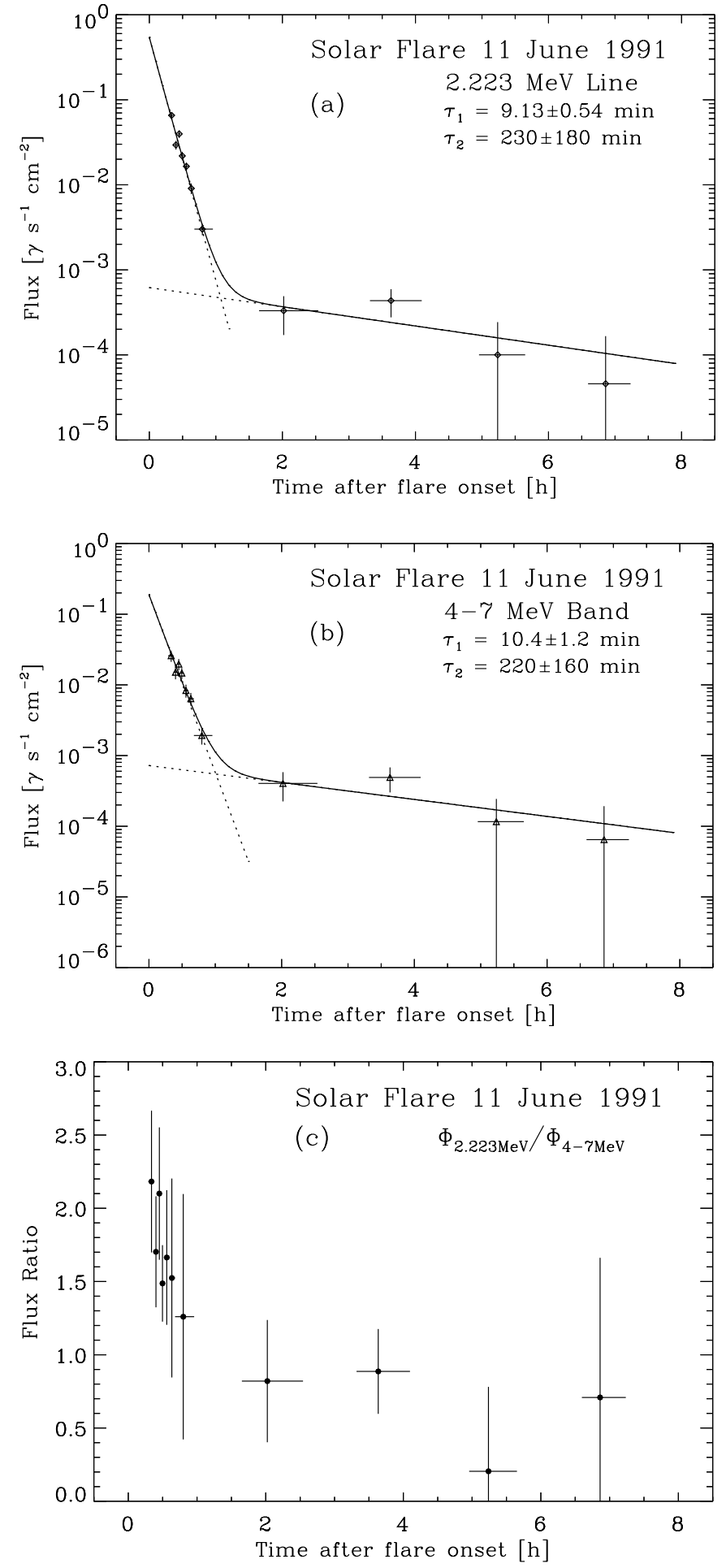

Fig. 6. Extended $\gamma$-ray emission as measured by COMPTEL for the 11 June 1991 flare in the $2.223 \mathrm{MeV}$ neutron capture line a), the 4-7 MeV nuclear line flux b), and their ratio c). The data have been corrected for primary and secondary bremsstrahlung. Error bars are $1 \sigma$ ranges, and horizontal bars denote the duration of the observation interval. A two-fold exponential decay has been fitted to the data and the best fit curve with the resulting decay times is included. The origin of the time axis is set to $0156 \mathrm{UT}$, i.e. the flare onset as reported by GOES. Only data of the extended phase (after 0213 UT) are displayed. 
defined in the neutron capture model (Fig. 5). We find $\Phi_{\text {brems }}=1371 \pm 100 \mathrm{ph} \mathrm{cm}^{-2}$ for the impulsive phase, $\Phi_{\text {brems }}=222 \pm 35 \mathrm{ph} \mathrm{cm}^{-2}$ for the intermediate phase, and $\Phi_{\text {brems }}=245 \pm 32 \mathrm{ph} \mathrm{cm}^{-2}$ for the extended phase. The ratio is $\Phi_{\text {brems }} / \Phi_{4-7}=16.0 \pm 2.6$ for the impulsive phase, $60 \pm 30$ for the intermediate phase, and $10.6 \pm 1.6$ for the extended phase.

The initial CGRO observation window ended at 0300 UT. During the following orbits, significant flux was still present in the $2.223 \mathrm{MeV}$ line and the $4-7 \mathrm{MeV}$ band. The emission lasted several hours, and is shown in Fig. 6 for the $2.223 \mathrm{MeV}$ line and the $4-7 \mathrm{MeV}$ range. For this figure, all instrumental corrections were done and bremsstrahlung contribution was subtracted, as described in Sect. 2.2. For the 8-30 MeV energy interval the emission falls below the sensitivity limit of COMPTEL. When data from all four consecutive orbits after the flare were combined, we determined an averaged flux of only $0.3 \pm 2.0 \gamma \mathrm{cm}^{-2}$. Hence, no significant signal can be detected in the COMPTEL data above $8 \mathrm{MeV}$ after $0300 \mathrm{UT}$.

The declining part of the extended emission in both the $2.223 \mathrm{MeV}$ line and the $4-7 \mathrm{MeV}$ emission can be described by two exponential decays. We performed a leastsquares fit of two exponential functions, and derived time constants for the $2.223 \mathrm{MeV}$ line of $9.13 \pm 0.54 \mathrm{~min}$ for the short and $230 \pm 180 \mathrm{~min}$ for the long decline constant. For the 4-7 MeV emission we computed $10.4 \pm 1.2 \mathrm{~min}$ for the short and $220 \pm 160 \mathrm{~min}$ for the long decline constants. For the short decay phase, the decay constants are somewhat shorter than the results obtained earlier from one single exponential fit. Some flux at the end of the short decay phase belongs already to the long decay component.

Figure 6c shows the ratio $\Phi_{2.2} / \Phi_{4-7}$ for the extended emission. The ratio tends to decrease slowly during the interval 2 to 7 hours after flare onset. However, the values for the last two orbits have a significance below $1 \sigma$ and the decrease of the ratio cannot be established firmly.

For the short-decay phase we determined fluences of $\Phi_{2.2 \mathrm{MeV}}=45.9 \pm 2.2 \gamma \mathrm{cm}^{-2}$ and $\Phi_{4-7} \mathrm{MeV}=23.1 \pm$ $1.8 \gamma \mathrm{cm}^{-2}$ with a ratio of $2.00 \pm 0.18$. For the long decay phase we find $\Phi_{2.2 \mathrm{MeV}}=2.6 \pm 0.8 \gamma \mathrm{cm}^{-2}$ and $\Phi_{4-7} \mathrm{MeV}=3.1 \pm 1.0 \gamma \mathrm{cm}^{-2}$ with a ratio of $0.84 \pm 0.37$.

All relevant fluence ratios for the different phases are summarized in Table 3.

\subsection{The solar flare on 9 June 1991}

The X10/3B flare on 9 June 1991 started at 0136 UT. The BATSE solar flare trigger was set at 0134:35 UT. This flare also started shortly after orbital sunrise. CGRO gathered data for more than one hour without interruption.

The impulsive phase of the flare started abruptly and produced severe instrumental dead time effects in the COMPTEL instrument. The dead time at the start of the flare was mainly caused by the limited telemetry capacity. Later, it was dominated by the high trigger rate of the

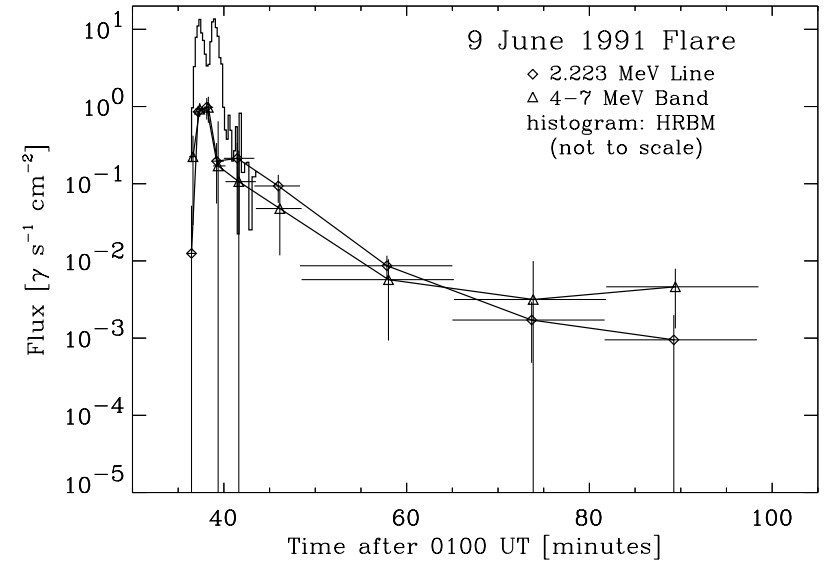

Fig. 7. Time profile for the 9 June 1991 flare. The fluxes in the $2.223 \mathrm{MeV}$ line and the $4-7 \mathrm{MeV}$ band were measured with the COMPTEL telescope, the HRBM counting rate reveals a double maximum of the $\gamma$-ray emission.

anti-coincidence detector system. The dead time reached a maximum of more than $98 \%$ during the impulsive phase of this flare. After about 0153 UT the dead time fell below the $50 \%$ level.

In Fig. 7 the deconvolved flux values for the $2.223 \mathrm{MeV}$ neutron capture line and the 4-7 MeV nuclear line emission between 0130 and 0245 UT are shown. Also, the count rate of the HRBM for the 4-7 MeV emission is included (not drawn to scale). The data reveal a pronounced double peak structure during the impulsive phase. In contrast to the 11 June 1991 flare, that was more gradual in its development, there are few data to analyze during the impulsive phase.

Beginning at about $0140 \mathrm{UT}$ the $2.223 \mathrm{MeV}$ and 4-7 MeV fluxes start to fall off monotonically. At the same time, the impulsive emission in the HRBM rate changes into a smoothly decaying curve. There is no such characteristic drop-off of the prompt emission after the impulsive phase as was observed for the 11 June 1991 flare.

Data during the impulsive phase (0136-0140 UT, $250 \mathrm{~s}$ duration) yield fluences of $\Phi_{2.2}=129 \pm 27 \gamma_{\mathrm{cm}^{-2}}$ and $\Phi_{4-7}=185 \pm 45 \gamma \mathrm{cm}^{-2}$. The ratio is $\Phi_{2.2} / \Phi_{4-7}=0.70 \pm$ 0.28 . For the energy range $8-30 \mathrm{MeV}$ the combined data yield a flux of $\Phi_{8-30}=1.7 \pm 4.4 \gamma \mathrm{cm}^{-2}$, equivalent to a significance of $0.4 \sigma$.

Similar to the 11 June flare, COMPTEL still detected a $\gamma$-ray signal during the orbits following the flare. In Fig. 8 the time profiles of the $2.223 \mathrm{MeV}$ line and the 4-7 MeV emission are shown. The first interval has a duration of about one hour. In subsequent orbits, the SAA interrupts the beginning of each orbital daytime pass. Therefore, the observations start later with each orbit and the observation intervals become progressively shorter.

The overall profile is similar to that of the 11 June flare. Again, a double exponential decay can describe the data. With the same fitting procedure we determined the time constants for the $2.223 \mathrm{MeV}$ line to be $4.9 \pm 0.9 \mathrm{~min}$ for the short and about $450 \mathrm{~min}$ for the long decay phase. 

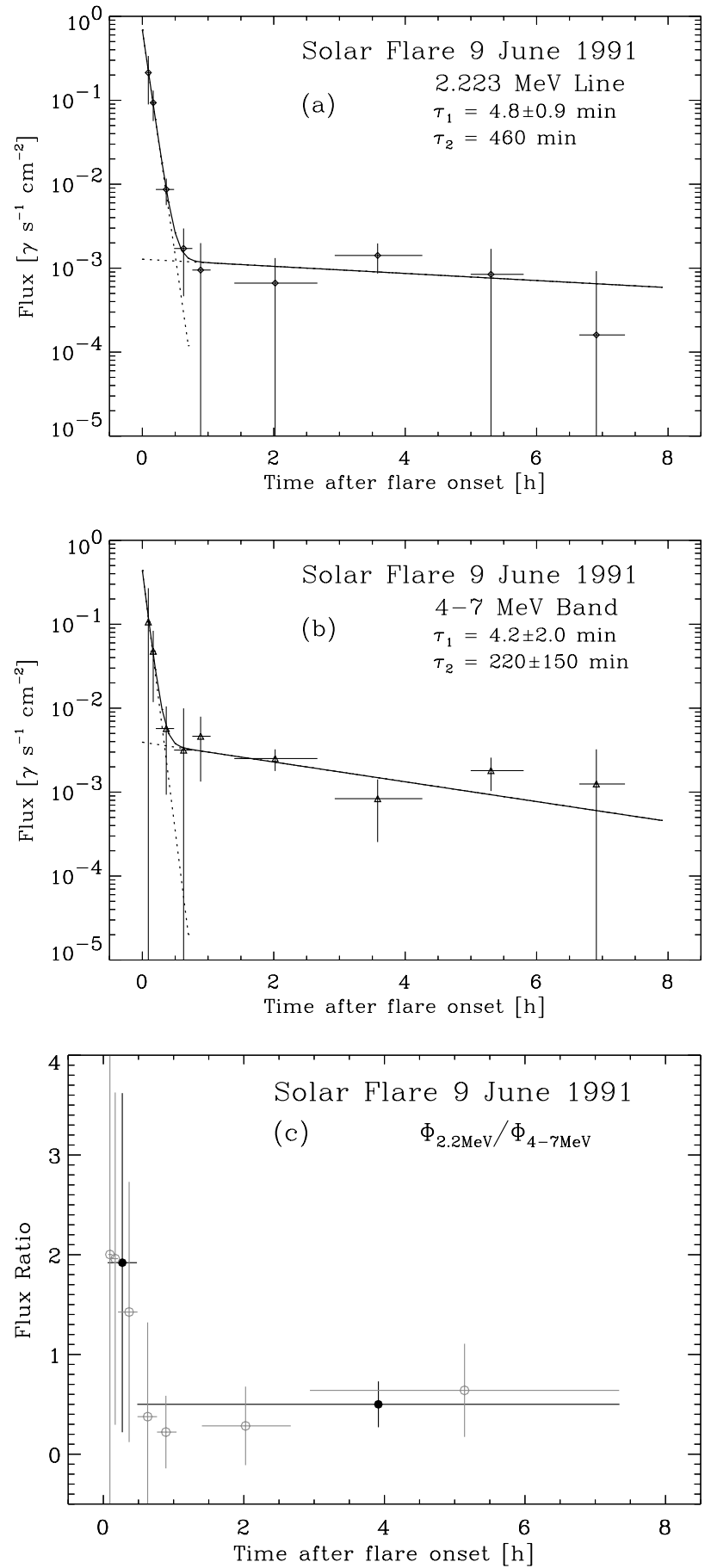

Fig. 8. Extended $\gamma$-ray emission for the 9 June 1991 flare in the $2.223 \mathrm{MeV}$ line a), the $4-7 \mathrm{MeV}$ nuclear line flux $\mathbf{b}$ ), and the ratio of both c). The data are plotted in the same way as in Fig. 6. In addition to the single data points (grey) we include the ratios for longer integration times (solid). The origin of the time axis has been set to a flare onset time of 0134 UT. Only data after 0140 UT are displayed.

The long decay, however, is consistent with a constant value. For the $4-7 \mathrm{MeV}$ emission we found $4.2 \pm 2.0 \mathrm{~min}$ for the short and $220 \pm 150 \mathrm{~min}$ for the long decay times.

During the impulsive phase we have found the $\Phi_{2.2} / \Phi_{4-7}$ ratio to be $0.70 \pm 0.28$. For later times we

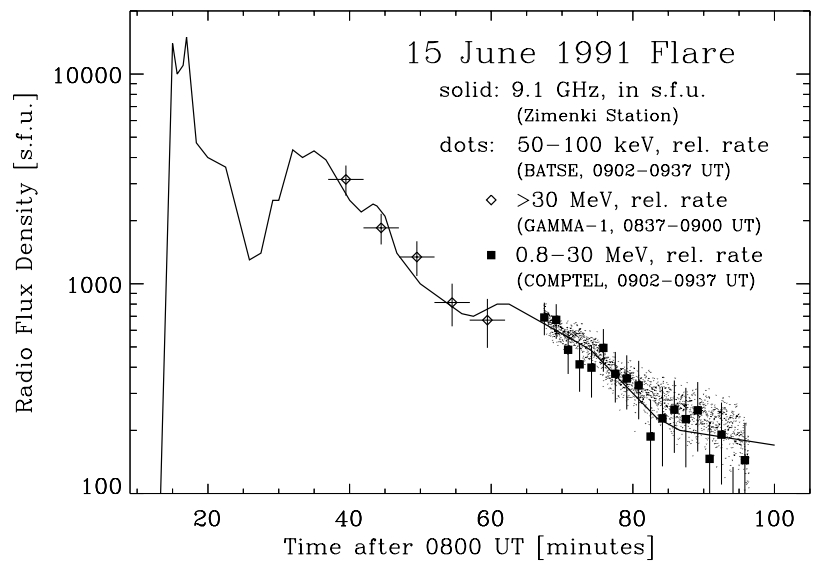

Fig. 9. Extended emission for the 15 June 1991 flare. Superimposed on the $9.1 \mathrm{GHz}$ microwave curve, given in solar flux units, s.f.u., are $\gamma$-ray measurements by GAMMA- 1 and COMPTEL, and HXR measurements by BATSE (microwave and GAMMA-1 data taken from Akimov et al. 1991; Kocharov et al. 1994).

computed a higher ratio of close to 2 that decreased over $30 \mathrm{~min}$ to a value below 1 . Individual data points do not yield statistically significant values for the fluence ratio. However, integrating the emission over the short and long decay phases, we determined the fluences for the short decaying component to be $\Phi_{2.2}=79.3 \pm 27.3 \gamma \mathrm{cm}^{-2}$ and $\Phi_{4-7}=41.3 \pm 34.5 \gamma \mathrm{cm}^{-2}$; the ratio being $1.92 \pm 1.70$. For the long decaying component we found $\Phi_{2.2}=6.2 \pm$ $2.1 \gamma \mathrm{cm}^{-2}$ and $\Phi_{4-7}=12.3 \pm 4.0 \gamma \mathrm{cm}^{-2}$; the ratio being $0.50 \pm 0.23$. In Fig. $8 \mathrm{c}$ the ratios for the time integrated fluences are plotted as solid data points while the ratios for the single measurements are plotted as grey data points. The statistical quality of the measurements does not preclude a constant value of $\Phi_{2.2} / \Phi_{4-7}$ for the whole period. The fluence ratios for the different phases are summarized in Table 3.

For all time intervals the $8-30 \mathrm{MeV}$ flux shows no excess above the primary bremsstrahlung continuum, indicating that pion decay radiation was not detected by COMPTEL.

\subsection{The solar flare on 15 June 1991}

The solar flare on 15 June 1991 was a 3B/X12 event and started at 0810 according to GOES-7 data. Active region NOAA 6659 was close to the west limb. No highenergy observations were conducted during the impulsive phase. However, there were measurements of radio and microwave emission. The GAMMA-1 instrument started observing the declining $\gamma$-ray signal from 0837 UT. It detected $\gamma$-rays from $50 \mathrm{MeV}$ up to about $1 \mathrm{GeV}$ for two observation periods (Akimov et al. 1991, 1994). CGRO exited the SAA at about 0900 UT and detected a $\gamma$-ray signal until Earth occultation (McConnell et al. 1993).

In Fig. 9 the time profile as measured by several instruments is shown. The impulsive phase was observed in 


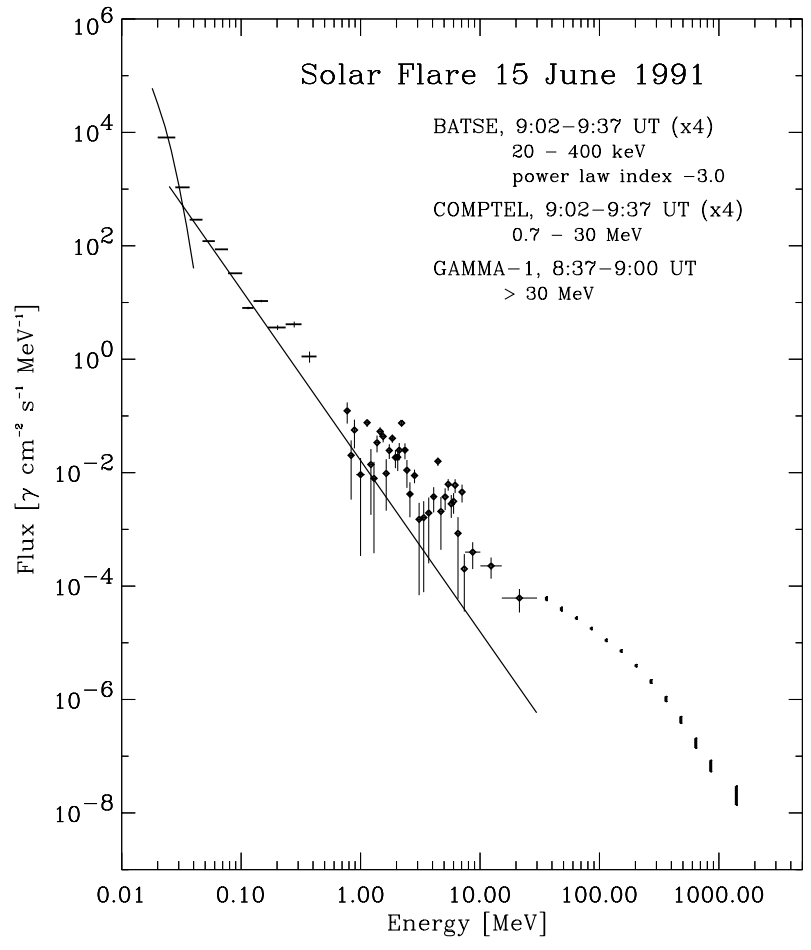

Fig. 10. Extended emission spectrum for the 15 June 1991 flare measured by different instruments.

9.1 GHz microwaves from Zimenki station (Kocharov et al. 1994). Displayed in arbitrary scaling are the count rates for $>30 \mathrm{MeV} \gamma$-rays detected by GAMMA-1 (Akimov et al. 1991), 1-30 MeV $\gamma$-rays detected by COMPTEL, and $50-100 \mathrm{keV}$ hard X-rays with a high time resolution of about $1 \mathrm{~s}$ detected by BATSE. Starting about $20 \mathrm{~min}$ after flare onset, the decline of the $\gamma$-ray flux at different energies tracks the microwave time profile. There is also a match between the HXR and microwave emission. Although both originate from energetic electrons, the agreement indicates that the microwaves are emitted from an optically thin region or at least from a region that is uniform and constant in opacity.

The results from GAMMA-1 and CGRO cannot be combined directly since the observation times do not overlap. However, since the $\gamma$-ray time profiles of both observations follow the $9.1 \mathrm{GHz}$ microwave emission (Fig. 9) we use the $9.1 \mathrm{GHz}$ time profile to normalize the $\gamma-$ ray flux measured by GAMMA-1 and CGRO relative to each other. After integrating the microwave curve over both observation periods we found that the microwave emission decreased by a factor of 4 from the earlier GAMMA-1 observation period to the subsequent CGRO period. Therefore, we scaled our derived spectra from BATSE and COMPTEL by a factor of 4 to join them with the GAMMA-1 spectrum at higher energies. The result is shown in Fig. 10. It shows the flare spectrum during the extended phase over five decades of energy from about $20 \mathrm{keV}$ to $2 \mathrm{GeV}$.
For the bremsstrahlung spectrum we fitted CONT data from BATSE with a single power law and a superhot thermal component. The resulting power law index is $3.00 \pm 0.05$ with a plasma temperature for the superhot component of about $45 \mathrm{MK}$. Both fit components and the data points used for the fit are shown in Fig. 10. The COMPTEL deconvolution is not affected by dead time and shows the $2.223 \mathrm{MeV}$ neutron capture line, and enhanced emission in the nuclear line range. Compared to the extended phase spectra of the 9 and 11 June 1991 flares (compare to Figs. 1 and 2), the $2.223 \mathrm{MeV}$ line is not as pronounced. The 15 June 1991 flare occurred close to the solar limb and the $2.223 \mathrm{MeV}$ line radiation could have been limb darkened. The continuum measured in the $8-30 \mathrm{MeV}$ range greatly exceeds the extrapolation from the bremsstrahlung power law. The spectrum measured by GAMMA-1 (Djantemirov et al. 1995) exhibits a steepening above about $100 \mathrm{MeV}$ indicating that the spectrum largely originates from the decay of charged and neutral pions. The transition between the spectra of COMPTEL below $30 \mathrm{MeV}$ and GAMMA-1 above $30 \mathrm{MeV}$ is smooth and supports the idea that the spectrum did not change significantly between the observation periods of GAMMA-1 and CGRO.

Our analysis revealed significant $\gamma$-ray emission for several hours in the $2.223 \mathrm{MeV}$ line, the 4-7 MeV band, and the $8-30 \mathrm{MeV}$ band. Figure 11 shows the long-term time profile of the flux measured by COMPTEL in the three components. The neutron capture line can be measured for about 6 hours. By fitting two exponential decay curves we find decay times of $10.3 \pm 2.0 \mathrm{~min}$ for the short and $180 \pm 110 \mathrm{~min}$ for the long decay phase. The emission in the $4-7 \mathrm{MeV}$ and $8-30 \mathrm{MeV}$ band is less significant and does not permit such a fit. If we combine the last three orbits (about 2.5 to 6 hours after flare onset) we determine a flux of $(2.81 \pm 0.88) \times 10^{-4} \mathrm{~cm}^{-2} \mathrm{~s}^{-1}$ for the $4-7 \mathrm{MeV}$ band, and $(2.04 \pm 0.90) \times 10^{-4} \mathrm{~cm}^{-2} \mathrm{~s}^{-1}$ for the $8-30 \mathrm{MeV}$ band, respectively. These combined values are included in Figs. 11b and $\mathrm{c}$ showing that COMPTEL indeed measures significant long-term emission in nuclear lines and pion-decay emission for as long as 6 hours after flare onset. In Figs. $12 \mathrm{a}$ and $\mathrm{b}$ the fluence ratios $\Phi_{2.2} / \Phi_{4-7}$ and $\Phi_{8-30} / \Phi_{4-7}$ are shown. We combined the last three observation periods as in Figs. $11 \mathrm{~b}$ and c. The ratio $\Phi_{2.2} / \Phi_{4-7}$ is consistent with a constant value of about 1 while the ratio $\Phi_{8-30} / \Phi_{4-7}$ may show a slow increase.

By integrating the fluxes during the short decay phase (up to $0925 \mathrm{UT}$ ) we computed fluences of $\Phi_{2.2}=4.51 \pm$ $0.52 \mathrm{~cm}^{-2}$ and $\Phi_{4-7}=5.08 \pm 0.75 \mathrm{~cm}^{-2}$ with a ratio of $0.88 \pm 0.15$. For the range $8-30 \mathrm{MeV}$ the fluence is $\Phi_{8-30}=1.6 \pm 0.3 \mathrm{~cm}^{-2}$ and the ratio $\Phi_{8-30} / \Phi_{4-7}$ is then $0.30 \pm 0.08$. Integrating the fluxes during the long decay phase yields $\Phi_{2.2}=2.41 \pm 0.65 \mathrm{~cm}^{-2}$ and $\Phi_{4-7}=2.54 \pm 0.77 \mathrm{~cm}^{-2}$ with a ratio of $0.95 \pm 0.38$. For the $8-30 \mathrm{MeV}$ band $\Phi_{8-30}=2.1 \pm 0.6 \mathrm{~cm}^{-2}$. The ratio is then $\Phi_{8-30} / \Phi_{4-7}=0.82 \pm 0.30$. Again, the fluence ratios for the different phases are summarized in Table 3. 

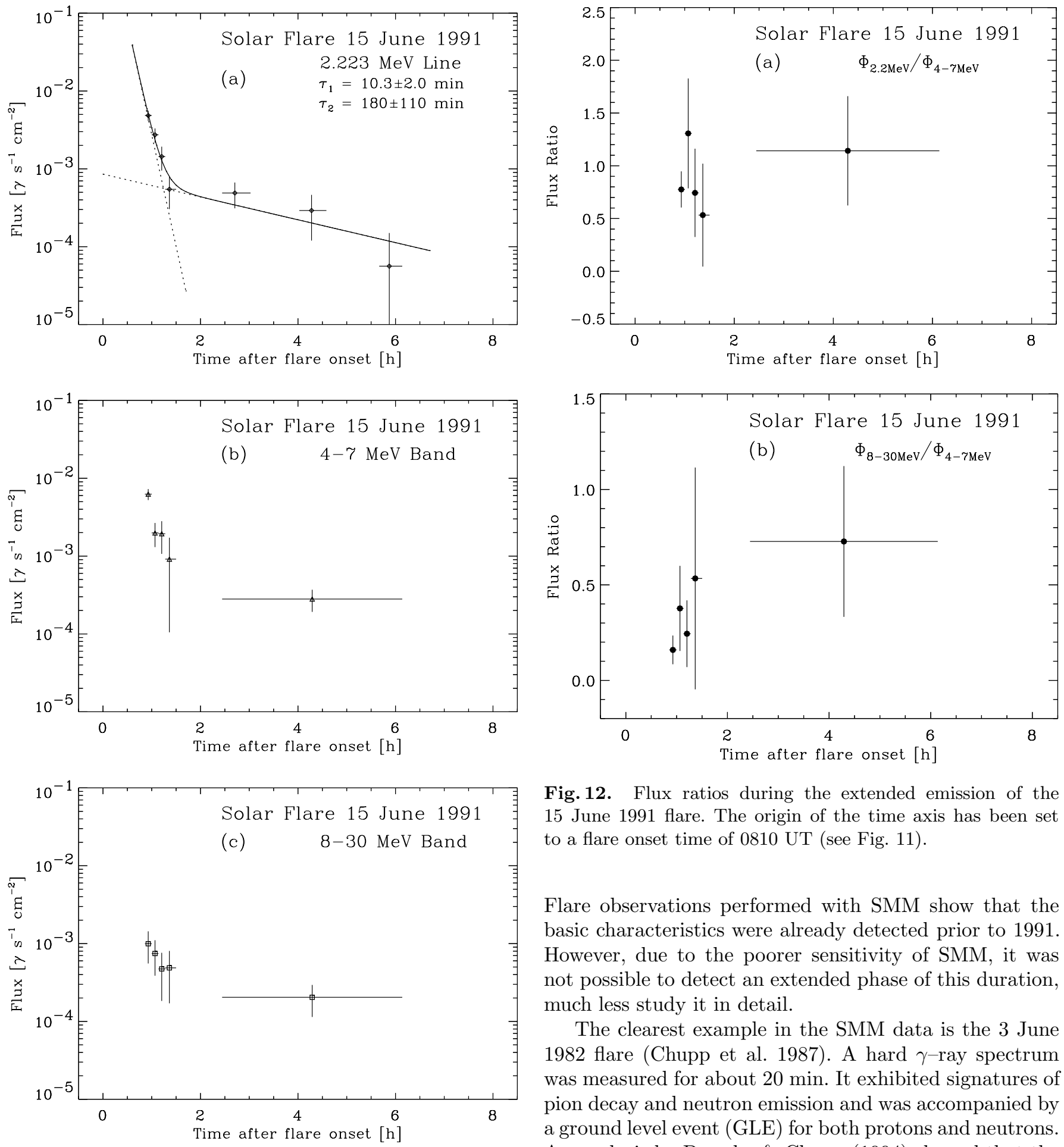

Fig. 11. Extended $\gamma$-ray emission for the 15 June 1991 flare in the $2.223 \mathrm{MeV}$ line a), the 4-7 MeV nuclear line flux b), and the $8-30 \mathrm{MeV}$ pion decay flux c). The data are plotted in the same way as in Fig. 6 . The origin of the time axis has been set to a flare onset time of $0810 \mathrm{UT}$. To improve the statistics of the datapoints in $\mathbf{b}$ ) and $\mathbf{c}$ ), the data for the last three orbits have been combined.

\section{Discussion}

The phenomenon of extended $\gamma$-ray emission for several hours has only been observed with instruments on CGRO.

Fig. 12. Flux ratios during the extended emission of the 15 June 1991 flare. The origin of the time axis has been set to a flare onset time of 0810 UT (see Fig. 11).

Flare observations performed with SMM show that the basic characteristics were already detected prior to 1991 . However, due to the poorer sensitivity of SMM, it was not possible to detect an extended phase of this duration, much less study it in detail.

The clearest example in the SMM data is the 3 June 1982 flare (Chupp et al. 1987). A hard $\gamma$-ray spectrum was measured for about $20 \mathrm{~min}$. It exhibited signatures of pion decay and neutron emission and was accompanied by a ground level event (GLE) for both protons and neutrons. An analysis by Dunphy \& Chupp (1994) showed that the $2.223 \mathrm{MeV}$ time profile of this flare could be fitted by two exponential decay curves with time constants of 1.15 and $11.7 \mathrm{~min}$.

Other examples of flares showing characteristics of extended emission are those on 16 December 1988 (Dunphy \& Chupp 1994), 24 May 1990 (Kocharov et al. 1994; Debrunner et al. 1997), and 26 March 1991 (Akimov et al. 1994).

All of these flares were large events with optical importance $2 \mathrm{~B}$ or greater, and GOES class X5 or greater. All had pronounced $\gamma$-ray line emission, and almost all 
of them exhibited pion decay and neutron emission. None could be identified as electron-rich. Interestingly, the signal from pion decay was usually recorded well after the impulsive phase and was accompanied by spectral hardening.

From COMPTEL data of the June 1991 flares we found that the general functional shape of the long-term time profiles during the extended phase are well described by two exponential decay curves. The time constants are on the order of $10 \mathrm{~min}$ for the short and a few $100 \mathrm{~min}$ for the long decay phase. The transition between both decay phases occurs about 1 to 2 hours after flare onset. The main parameters of the extended emission time profiles determined by our analysis from COMPTEL data are summarized in Table 2.

Not only was a two-fold exponential decay curve of the extended emission found in the $2.223 \mathrm{MeV}$ line for all three flares on 9, 11 and 15 June (compare Figs. 8a, 6a, and 11a), but the same profile was found in the $4-7 \mathrm{MeV}$ band for the 9 and 11 June flare (Figs. 8b, and 6b). The 4-7 MeV profile of the 15 June flare does not suggest by itself a double exponential decay but is well consistent with it (Fig. 11b). The pion decay flux during the short decay phase of the extended emission of the 11 June 1991 flare decreases, analogous to the prompt 4-7 MeV emission and the $2.223 \mathrm{MeV}$ line (Fig. 4a). Well into the extended phase COMPTEL did not detect a $8-30 \mathrm{MeV}$ signal. Measurements with the EGRET spark chamber, however, show a $>50 \mathrm{MeV}$ signal for up to 11 hours also displaying a two-fold exponential decay curve (Kanbach et al. 1993). Our non-detection of the long-term 8-30 MeV emission after 0300 UT is consistent with the poorer sensitivity of COMPTEL at these energies. For the 15 June event, the profile of the $8-30 \mathrm{MeV}$ band measured by COMPTEL (Fig. 11c) does not require a double exponential decay, but is consistent with it, given that no measurements were made during the first $40 \mathrm{~min}$ after flare onset. Measurements of the $>50 \mathrm{MeV}$ radiation with the GAMMA-1 instrument at earlier times between about 0837 and 0900 UT indicate an exponential decay with a decay time of about $9.8 \mathrm{~min}$ (Akimov et al. 1991; Kocharov et al. 1994). Emission $>50 \mathrm{MeV}$ was reported also for the following observation period of GAMMA-1 from 1010 to 1040 UT (Akimov et al. 1994). For the 9 June flare no signal from pion decay could be detected by COMPTEL at any time. Consistent with these results, Murphy et al. (1997) found a comparable decay profile consisting of two different exponential decline constants for the 4 June 1991 flare from OSSE data.

By comparing the properties of the flares on 9,11 and 15 June 1991 the similarity of the long-term time profiles is conspicuous. In particular, the time profiles of the $2.223 \mathrm{MeV}$ line emission are surprisingly similar. In Fig. 13 we have plotted the $2.223 \mathrm{MeV}$ profiles of all three flares normalized to the time and flux of the transition from the short to the long decay phase, according to Table 2. Although the data come from three different flares several days apart, the profiles are identical within statistics.

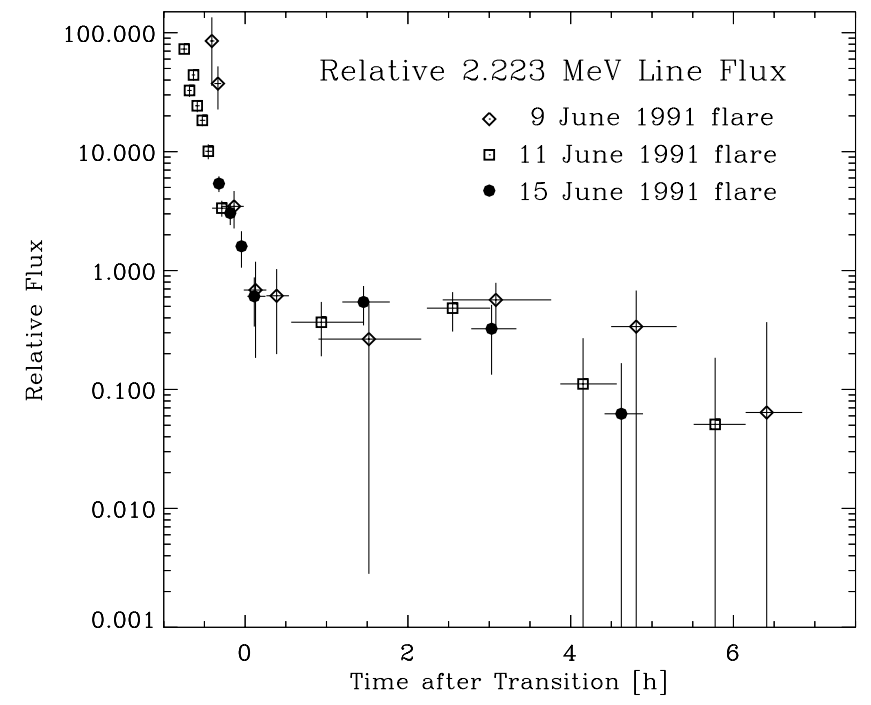

Fig. 13. Relative time profiles of the $2.223 \mathrm{MeV}$ neutron capture line for the flares on 9, 11, and 15 June 1991. The origin of the plot has been set to the time and flux level of the transition, $T_{\mathrm{t}}$ and $\Phi_{\mathrm{t}}$, according to Table 2 . Only data during the extended phase are included.

Our ability to answer the question whether the emission during the extended phase is smoothly declining or if it is accompanied by bursts of new emission relies upon the sensitivity of the instrument. For the COMPTEL telescope we had to integrate over $\approx 100 \mathrm{~s}$ during the short decay phase, and $\approx 2000 \mathrm{~s}$ (the whole orbital daytime periods of GCRO) during the long decay phase. On these scales, the emission varies smoothly and does not show any fine structure or significant bursts.

During the first hour of the 11 June flare there is one time interval (at about 0223 UT, Fig. 4a) that shows enhanced emission in both the $2.223 \mathrm{MeV}$ line and the 4-7 MeV band, but not in the $8-30 \mathrm{MeV}$ band. The HXR data of BATSE also show enhanced emission from $25 \mathrm{keV}$ to $300 \mathrm{keV}$ (Fig. 3a). This burst is superimposed on a relatively smooth decay. The peak in the HXR emission was accompanied by a spectral softening that can be seen as an increase in the bremsstrahlung power law index in Fig. 3b. The increase in emission can also be identified in the Nobeyama $17 \mathrm{GHz}$ microwave data (Mandzhavidze et al. 1996).

Another interesting feature can be found in the data during the long decay phase of the extended emission. After the transition from short to long decay the emission first rises for about 1 to 2 hours, decreasing afterwards. This behaviour can be seen for the 11 June flare in the $2.223 \mathrm{MeV}$ line (Fig. 6a), and the $4-7 \mathrm{MeV}$ band (Fig. 6b), for the 9 June flare in the $2.223 \mathrm{MeV}$ line (Fig. 8a), and for the 15 June flare in the $2.223 \mathrm{MeV}$ line (Fig. 11a). The same trend appears in all three flares suggesting that the increase is real. The increasing flux at the beginning of the long decay phase is particularly intriguing in the combined plot of the $2.223 \mathrm{MeV}$ flux in Fig. 13. 
Table 2. Characteristic parameters for the extended phase.

\begin{tabular}{llllll}
\hline flare & energy & $\tau_{1}^{\mathrm{a}}$ & $\tau_{2}^{\mathrm{a}}$ & $\Phi_{\mathrm{t}}^{\mathrm{b}}$ & $T_{\mathrm{t}}^{\mathrm{c}}$ \\
\hline 9 June & $2.2 \mathrm{MeV}$ & $4.9 \pm 0.9 \mathrm{~min}$ & $450 \mathrm{~min}$ & $2.5 \times 10^{-3}$ & $30 \mathrm{~min}$ \\
& $4-7 \mathrm{MeV}$ & $4.2 \pm 2.0 \mathrm{~min}$ & $220 \pm 150 \mathrm{~min}$ & $6.5 \times 10^{-3}$ & $20 \mathrm{~min}$ \\
11 June & $2.2 \mathrm{MeV}$ & $9.13 \pm 0.54 \mathrm{~min}$ & $230 \pm 180 \mathrm{~min}$ & $9.0 \times 10^{-4}$ & $65 \mathrm{~min}$ \\
& $4-7 \mathrm{MeV}$ & $10.4 \pm 1.2 \mathrm{~min}$ & $220 \pm 160 \mathrm{~min}$ & $1.0 \times 10^{-3}$ & $60 \mathrm{~min}$ \\
& pion decay & $10.8 \pm 1.4 \mathrm{~min}$ & & & \\
& bremsstr. & $8.6 \pm 1.0 \mathrm{~min}$ & & & $75 \mathrm{~min}$ \\
\hline \hline
\end{tabular}

a $\tau_{1}, \tau_{2}$ : decay constants for the short and long decay phase, respectively;

b $\Phi_{t}$ : photon flux $\left[\gamma \mathrm{cm}^{-2} \mathrm{~s}^{-1}\right]$ at which the transition occurs;

c $T_{\mathrm{t}}$ : time after flare onset at which the transition occurs.

All these peculiarities demonstrate that the decline of the extended emission is not monotonic but is accompanied by episodes of enhanced flux, that occur simultaneously in both the X-ray and nuclear $\gamma$-ray emission.

We also investigated the spectral development of the flares. We analyzed the fluence ratio between the $2.223 \mathrm{MeV}$ neutron capture line and the 4-7 MeV prompt nuclear line emission. If the integration time is long enough, this can be used as an indicator of the spectral hardness of the accelerated protons and ions in the energy range of about $10-50 \mathrm{MeV}$. We compare our observational results to theoretical results derived by Ramaty et al. (1993) to convert the ratio $\Phi_{2.2} \mathrm{MeV} / \Phi_{4-7} \mathrm{MeV}$ into a spectral hardness parameter. Since the $2.223 \mathrm{MeV}$ photons are attenuated for flares near the solar limb, the calculations were performed for different heliocentric angles and different angular distributions of the interacting particles.

Ramaty et al. (1993) studied two sets of elemental abundances: photospheric abundances for both beam and target (composition 1), and abundances as determined by Murphy et al. (1990) for the 27 April 1981 flare (composition 2) which are enhanced in $\alpha$ particles and heavy elements. Here we assume that particles interact isotropically in the solar atmosphere with composition 1 . If either a horizontally interacting particle population or composition 2 is used the resulting spectrum will be harder.

For the 11 and 15 June 1991 flares, we also computed the flux ratio $\Phi_{8-30 \mathrm{MeV}} / \Phi_{4-7} \mathrm{MeV}$ that is sensitive to the accelerated particle spectrum at a few hundred $\mathrm{MeV}$. For the 9 June 1991 flare we did not detect a pion decay signal. Since the COMPTEL energy range is limited to $<30 \mathrm{MeV}$ we only measured a fraction of the entire pion decay flux and, hence, the ratio can only be used to indicate spectral changes. Additionally, the spectrum of the accelerated electrons was analyzed from HXR data for the 11 June 1991. From all available spectral diagnostics including the HXR data for the 11 June 1991 flare we find that there are significant changes in the emission characteristics and the accelerated particle spectra during the June 1991 flares.

The most complete picture of extended $\gamma$-ray emission can be drawn from the 11 June 1991 flare. According to the variations in the accelerated particle spectrum, the HXR spectrum, and differences in the neutron capture time, we divide the flare into three different parts that we call the impulsive, intermediate and extended phases:

Impulsive phase: The impulsive phase (0158-0207 UT, denoted as part I in Fig. 5) exhibits a soft accelerated particle spectrum with power law index of about -4.3 assuming isotropically interacting particles and composition 1 . The emission measured at $8-30 \mathrm{MeV}$ is consistent with the extrapolated primary bremsstrahlung continuum. This suggests that no significant production of pions took place during the impulsive phase. Only an upper limit can be assigned to the ratio $\Phi_{8-30} \mathrm{MeV} / \Phi_{4-7} \mathrm{MeV}$. The electron bremsstrahlung spectrum measured by BATSE can be described by a broken power law early in the impulsive phase until the instrument dead time invalidates the data. PHEBUS which was not limited by dead time effects measured the bremsstrahlung continuum above $75 \mathrm{keV}$. The PHEBUS power-law index is consistent with that found from BATSE above the break energy. It did not appear to change during the entire impulsive phase. The fluence of the integrated bremsstrahlung $>300 \mathrm{MeV}$ and the $4-7 \mathrm{MeV}$ fluence have a ratio of $16.0 \pm 2.6$. During the impulsive phase the emission is well structured and shows clearly defined peaks. Modeling the neutron capture time profile yields a neutron capture time delay $\lambda$ of $(65 \pm 10) \mathrm{s}$, consistent with the value of $(70 \pm 10)$ s determined by Trottet et al. (1993) from PHEBUS data, and $(95 \pm 40) \mathrm{s}$ determined by Dunphy et al. (1999) from EGRET data for about the same time period. The measured capture time delay $\lambda$ is related to the hydrogen density $n(\mathrm{H})$ in $\mathrm{cm}^{-3}$ in the capture region and the ratio $r=n\left({ }^{3} \mathrm{He}\right) / n(\mathrm{H})$ by

$\frac{1}{\lambda}=\frac{1}{\lambda_{\mathrm{d}}}+\frac{n(\mathrm{H})}{1.4 \times 10^{19}}+\frac{r \times n(\mathrm{H})}{8.5 \times 10^{14}}$ 
where $\lambda_{\mathrm{d}}=917 \mathrm{~s}$ is the mean neutron life time for spontaneous decay. Assuming that the hydrogen density of $n(\mathrm{H})=1.3 \times 10^{17} \mathrm{~cm}^{-3}$ does not to vary significantly within the capture region (Prince et al. 1983; Hua \& Lingenfelter 1987) we can use Eq. (3) to determine the ${ }^{3} \mathrm{He} / \mathrm{H}$ ratio. We find $r=(3.3 \pm 1.6) \times 10^{-5}$. This value is close to the result of $3.0 \times 10^{-5}$ deduced by Trottet et al. (1993). Abundance ratios in the same range have been reported for other flares, e.g., $r=5.0 \times 10^{-5}$ for the 7 June 1980 flare (Chupp et al. 1981), $r=2.3 \times 10^{-5}$ for the 3 June 1982 flare (Hua \& Lingenfelter 1987), and $r=1.3 \times 10^{-5}$ for the 4 June 1991 flare (Murphy et al. 1997).

Intermediate phase: After the impulsive phase the prompt 4-7 MeV emission fades quickly while the $2.223 \mathrm{MeV}$ line lingers. An understanding of this intermediate phase (0205-0213 UT, part II in Fig. 5) is incomplete at this point. The high $\Phi_{2.2} \mathrm{MeV} / \Phi_{4-7} \mathrm{MeV}$ ratio indicates a hard spectrum, suggesting a strong pion decay emission that is not observed. If the emission is explained as delayed neutron capture this would require neutrons to reside in the photosphere for more than $5 \mathrm{~min}$. This is a significant fraction of the spontaneous decay time $\lambda_{\mathrm{d}}$. An unusually long delay of the photospheric neutron capture is found by modeling the $2.223 \mathrm{MeV}$ line emission. We determined the capture time to be $\lambda=230 \mathrm{~s}$ with a $1 \sigma$ range of $140-$ $390 \mathrm{~s}$. However, even under the assumption of a vanishing ${ }^{3} \mathrm{He}$ abundance the capture time $\lambda$ for a hydrogen density of $n(H)=1.3 \times 10^{17} \mathrm{~cm}^{-3}$ cannot be much greater than $100 \mathrm{~s}$. Our result instead suggests that the neutrons are captured in a layer higher up in the photosphere where the hydrogen density is accordingly lower. To explain a value of $\lambda=140 \mathrm{~s}$, the lower limit of our $1 \sigma$ confidence range, we derive $n(H)=8.5 \times 10^{16} \mathrm{~cm}^{-3}$ if we assume a vanishing ${ }^{3} \mathrm{He}$ abundance $(r=0)$, and a hydrogen density $n(H)$ of $5.5 \times 10^{16} \mathrm{~cm}^{-3}$, for a normal value of $r=3 \times 10^{-5}$ (Hua \& Lingenfelter 1987). A somewhat large value for the neutron capture time of $104 \pm 6 \mathrm{~s}$ has also been found for the 4 June 1991 flare from OSSE data (Murphy et al. 1997). The conditions for neutron capture taking place in a region with very low hydrogen density might be met if either the neutrons are low in energy, hence not being able to penetrate deep into the photosphere before being thermalized, or they preferrently enter the photosphere at a small angle thermalizing in a shallow photospheric layer.

Extended phase: A strong nuclear line signal is measured again at $0213 \mathrm{UT}$, about $15 \mathrm{~min}$ after the onset of the impulsive phase. We identify this time as the onset of the extended phase. During the following $40 \mathrm{~min}$ until the end of the orbital period the accelerated particle spectrum is harder than that of the impulsive phase. For the period from 0213 to $0303 \mathrm{UT}$ we deduce a power law index of 3.7 (isotropic particle interactions, composition 1). However, according to the hardness ratio plotted in Fig. 4b the proton spectrum becomes softer afterwards. Hours after flare onset the spectral hardness is comparable to that of the impulsive phase (Fig. 6c). This trend could not be confirmed with the neutron capture model (Fig. 5) since the HRBM data become very noisy after about 0220 UT. Beginning with the onset of the extended phase a strong excess signal above $8 \mathrm{MeV}$ appears above the primary bremsstrahlung continuum. We attribute this signal to pion decay emission, indicating that during the extended phase protons are accelerated to energies of several $100 \mathrm{MeV}$. The ratio $\Phi_{8-30} \mathrm{MeV} / \Phi_{4-7} \mathrm{MeV}$ is $0.62 \pm 0.08$ and holds throughout the extended phase. The hard X-ray spectra measured by BATSE are well described by a single power law with an additional superhot thermal component. The measured plasma temperature of the superhot component increases from about $20 \mathrm{MK}$ to $60 \mathrm{MK}$ between 0213 and $0230 \mathrm{UT}$, and then slowly decreases. The bremsstrahlung spectrum in the $100 \mathrm{keV}$ range has a power law index of about 3.5 as compared to about 2.5 during the impulsive phase. During the extended phase from 0213 to 0300 UT the power law index remains unchanged within errors (compare to Fig. 3b). We note that the electron spectrum softens as the flare develops from the impulsive to the extended phase, while the proton spectrum hardens. This suggests that during the impulsive phase the conditions for electrons to reach high energies are favourable, while the same is true for the protons during the extended phase. In general, during this period the emission appears to be more gradual than during the impulsive phase. The ratio of the integrated bremsstrahlung $>300 \mathrm{keV}$ and the $4-7 \mathrm{MeV}$ fluence is $10.6 \pm 1.6$. This value is significantly lower than during the impulsive phase and implies that the $\mathrm{e} / \mathrm{p}$ ratio of the accelerated particles has decreased.

The boundaries between the phases have been determined by the modeling of the time profile of the neutron capture line using the HRBM data. The model is sensitive to changes in the hardness ratio and/or the neutron capture time delay and the boundaries can be determined to within one minute. The same division can be found with less precision using the ratios $\Phi_{2.2} / \Phi_{4-7}$ and $\Phi_{8-30} / \Phi_{4-7}$. A separation of the 11 June 1991 flare into different phases was already suggested by Mandzhavidze et al. (1996), Rank et al. (1997), and Dunphy et al. (1999). Here, we use similar criteria for the distinction of the phases and the same subdivisions as Rank et al. (1997).

Mandzhavidze et al. (1996) studied $>50 \mathrm{MeV}$ data from the EGRET spark chamber, $2.223 \mathrm{MeV}$ data from COMPTEL and $17 \mathrm{GHz}$ microwave data from Nobeyama. The time constants they found for the $2.223 \mathrm{MeV}$ line, the pion decay emission, and the $17 \mathrm{GHz}$ data during the extended phase differ from each other. The $17 \mathrm{GHz}$ data show an enhancement at the beginning of the long decay phase that is interpreted as new particle acceleration. Also, a spectral hardening from the impulsive to the extended phase, and indications for a softening of the proton spectrum late in the extended phase have been reported.

From analyzing EGRET/TASC data Dunphy et al. (1999) defined three phases that are similar to those defined by Rank et al. (1997) and the present analysis. They also found a hardening of the accelerated proton spectrum from the impulsive to the extended phase. 
Table 3. Spectral hardness for different components.

\begin{tabular}{cccccccc}
\hline flare & interval & phase $^{\mathrm{a}}$ & $\Phi_{2.2} / \Phi_{4-7}$ & $s^{\mathrm{b}}$ & $\alpha T^{\mathrm{b}}$ & $\Phi_{8-30} / \Phi_{4-7}$ & $\Phi_{\text {brems }} / \Phi_{4-7}$ \\
\hline \multirow{2}{*}{ 9 June } & $0136-0140$ & imp & $0.70 \pm 0.28$ & $4.5 \pm 0.4$ & $0.010 \pm 0.004$ & - & - \\
& $0140-0205$ & ext1 & $1.92 \pm 1.70$ & $\approx 3.6$ & $\approx 0.017$ & - & - \\
& after 0205 & ext2 & $0.50 \pm 0.23$ & $\approx 4.8$ & $\approx 0.008$ & - & - \\
11 June & $0158-0207$ & imp & $0.82 \pm 0.15$ & $4.3 \pm 0.3$ & $0.011 \pm 0.003$ & $<0.04$ & $16.0 \pm 2.6$ \\
& $0207-0213$ & int & $5.6 \pm 2.5$ & $\approx 2.7$ & $\approx 0.06$ & $<0.20$ & $60 \pm 30$ \\
& $0213-0303$ & ext1 & $2.00 \pm 0.18$ & $3.7 \pm 0.3$ & $0.017 \pm 0.004$ & $0.62 \pm 0.08$ & $10.6 \pm 1.6$ \\
& after 0303 & ext2 & $0.84 \pm 0.37$ & $\approx 4.3$ & $\approx 0.011$ & - & - \\
15 June & $0900-0925$ & ext1 & $0.88 \pm 0.15$ & $3.7 \pm 0.3$ & $0.017 \pm 0.004$ & $0.30 \pm 0.08$ & - \\
& after 0525 & ext2 & $0.95 \pm 0.38$ & $3.6 \pm 0.5$ & $0.018 \pm 0.008$ & $0.82 \pm 0.30$ & - \\
\hline
\end{tabular}

a imp: impulsive phase; int: intermediate phase; ext1, ext2: short and long decay phase.

b $s, \alpha T$ : power law index, Bessel function parameter according to Ramaty et al. (1993).

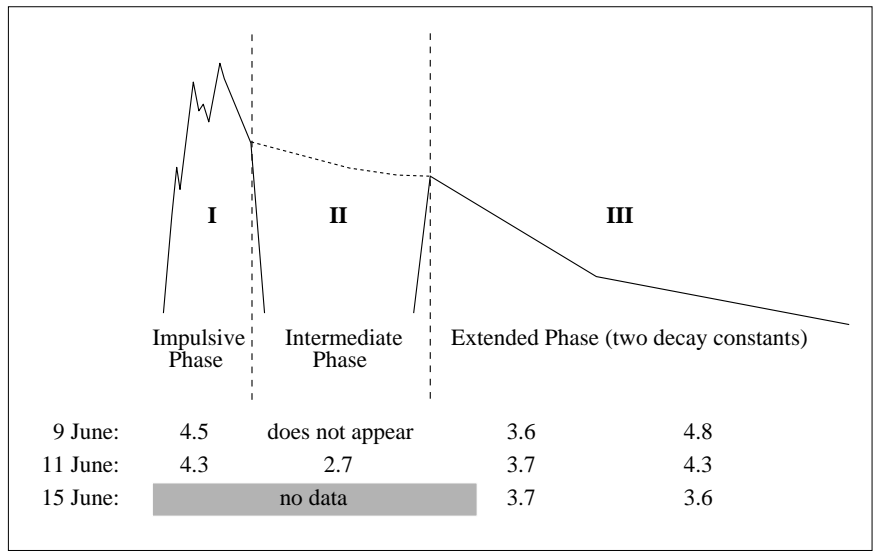

Fig. 14. Sketch of the spectral changes during the extended phase of the June 1991 flares. For each phase which we identified the power law index of the accelerated proton spectrum is given (see text for details).

Their value for the $\Phi_{2.2} / \Phi_{4-7}$ ratio during the impulsive phase of $1.28 \pm 0.12$ is significantly higher than our value of $0.82 \pm 0.15$. The reason for this difference could be that Dunphy et al. (1999) integrated over a longer time interval at the end of the impulsive phase where the $2.223 \mathrm{MeV}$ flux is much greater than the $4-7 \mathrm{MeV}$ flux. During the extended phase between 0213 and 0300 UT the ratios of $\Phi_{2.2} / \Phi_{4-7}=1.82 \pm 0.18$ as reported by Dunphy et al. (1999) and $2.00 \pm 0.18$ as found here agree with each other. Although the sensitivity range of the TASC extends to higher energies it detected no pion decay signal before the onset of the extended phase, consistent with our results. Dunphy et al. (1999) derived an upper limit for the impulsive phase of $\Phi_{\text {pion }}<0.107 \mathrm{~cm}^{-2}$, more restrictive than $\Phi_{8-30}<2.0 \mathrm{~cm}^{-2}$ from COMPTEL data.

Both Mandzhavidze et al. (1996) and Dunphy et al. (1999) noted that after the impulsive phase the prompt emission fades away while the $2.223 \mathrm{MeV}$ line is still present. They suggest that this is due to the delayed nature of the neutron capture process. As mentioned before, this interpretation is problematic since it requires the abundance of free neutrons for an unprecedented time span.

There appears to be an important difference between the transition from the impulsive to the extended phase, and from the short to the long decay phase during the extended emission. Besides the clear change in the time constants, our data suggest that any spectral changes are subtle and occur slowly and gradually. On the contrary, the changes from phase I to II at 0207 UT, and II to III at 0213 UT (Fig. 14) occur abruptly, and are accompanied by further parameter changes, such as neutron capture time delay, and pion decay emission. For this reason we suggest that the short and long decay phases are not fundamentally different. The observation of two different decay constants may be easier to explain by the change from one particle population dominating the emission to another.

We have studied the development of the 11 June 1991 flare in closer detail and have found a hardening of the accelerated proton and ion spectrum from the impulsive to the extended phase. During the extended phase the spectrum tends to soften gradually.

Basically the same variations appear during the 9 June 1991 flare. Starting with a power law index of 4.5 for the accelerated particle spectrum during the impulsive phase we found a power law spectral index of about 3.6 during the short decay phase of the extended emission. Afterwards, the spectrum became softer and for the long decay phase we found an index of 4.8 for the entries phase. No feature comparable to the intermediate phase of the 11 June 1991 flare could be identified here. For the 9 June 1991 event it appears as if the extended phase sets in before the emission from the impulsive phase has subsided. Furthermore, the duration of the impulsive phase, and the time between flare onset and phase transition are much shorter for the 9 June 1991 flare than those for the 11 June 1991 flare. 
For the 15 June 1991 flare the impulsive phase was not observed. Interestingly, however, the measured profile for the $9.1 \mathrm{GHz}$ microwave emission (Fig. 9) resembles in many ways the 11 June 1991 flare $\gamma$-ray emission: a structured impulsive phase, a phase of very low emission, and a more gradually varying long-term emission. Contrary to the other two flares, the spectral shape during the extended phase does not show any significant change, as measured by the $\Phi_{2.2} / \Phi_{4-7}$ and $\Phi_{8-30} / \Phi_{4-7}$ ratios. By analyzing the $\Phi_{2.2} / \Phi_{4-7}$ ratio we are only able to determine a power law index for the accelerated particle spectrum valid over the range of about $10-100 \mathrm{MeV} /$ nucleon. By combining data from COMPTEL and GAMMA-1 Kocharov et al. (1998) deduced the shape of the accelerated particle spectrum for the short component of the 15 June 1991 flare over a much wider energy range. For the proton spectrum, they deduced a power law index of 3.3, with a steepening at about $1 \mathrm{GeV}$. At high energies an additional component with an index of about 3.0 is necessary.

The changes we see in several flux ratios for the three flares in June 1991 are summarized in Table 3. We computed the index s for a power law and the spectral parameter $\alpha \mathrm{T}$ for the accelerated particle spectrum for isotropic interactions and composition 1 of Ramaty et al. (1993). They are deduced from the ratio $\Phi_{2.2} / \Phi_{4-7}$ and are valid for the energy range of about $10-100 \mathrm{MeV} /$ nucleon. Figure 14 is a diagram of the spectral changes and the power law indices according to Table 3 of the three flares studied.

Besides many similarities between the flares on 9, 11 and 15 June 1991 there are also differences. One is the behaviour of the pion decay radiation for each of the flares. In the COMPTEL data, the pion decay signal is completely absent during the 9 June 1991 flare. For the 11 June flare, strong pion decay emission was detected with COMPTEL during the first $30 \mathrm{~min}$ of the extended phase. We did not detect a significant signal afterwards, but from the EGRET measurements we know that the pion decay flux persisted for more than 10 hours. In the case of the 15 June flare, strong pion decay emission is seen with COMPTEL throughout the observation which lasted 5 hours after flare onset. On the other hand we find the time profiles of the $2.223 \mathrm{MeV}$ line (compare Fig. 13) and the $4-7 \mathrm{MeV}$ band very similar. This shows that in each of the flares protons with about $10-100 \mathrm{MeV}$ are present in comparable numbers and distribution, and also with a comparable time profile. On the other hand a larger number of pions was generated for each successive flare. This suggests that the acceleration of particles up to high energies differs from flare to flare.

Our measurements as well as the results from other instruments establish the fact that high-energetic protons and ions are present for several hours throughout the extended phase. Mandzhavidze \& Ramaty (1992) proposed that particles are accelerated during the impulsive phase and are subsequently trapped in closed magnetic loop structures. The slow leakage of particles at the footpoints causes the prolonged $\gamma$-ray emission. According to Ryan \& Lee (1991) the particles are continuously accelerated during the extended phase in turbulent loop structures and precipitate at the loop footpoints. Both models are similar in the sense that the particles need to be trapped in closed structures for several hours. However, in the first case the confinement of energetic particles is realized by magnetic mirroring near the footpoints of coronal loops ("magnetic trapping"). In the second case, the particles which are still gaining energy must be confined efficiently enough so that they can reach high energies. Intense plasma turbulence is necessary to provide efficient scattering of the particles, providing both the confinement ("collisional trapping") and the source of energy. Akimov et al. (1991) also suggested a model of continuous acceleration, but in a current sheet that forms in the wake of a rising CME. We will now confront the proposed models with our measurements for the June 1991 flares.

In the modeling done for the EGRET $>50 \mathrm{MeV}$ measurements of the 11 June 1991 flare (Mandzhavidze \& Ramaty 1992; Ramaty \& Mandzhavidze 1994), and the GAMMA-1 and COMPTEL measurements of the 15 June 1991 flare (Mandzhavidze et al. 1993), the level of plasma turbulence must be very low and allow only few particles to be removed from the trap to accomplish the long precipitation times. The model assumes a loop size of $10^{7} \mathrm{~m}$ and requires a very low energy density of the turbulence of $<2 \times 10^{-8} \mathrm{erg} \mathrm{cm}^{-3}$, as well as a high mirror ratio between the loop footpoints and the coronal part of $B_{\max } / B_{\min }=50$ where $B_{\max }<10 \mathrm{G}$ in the coronal part of the loop. Twisting of the loops reduces the rate of particles being removed from the trap by drift (Lau \& Ramaty 1994; Ramaty \& Mandzhavidze 1996) and help to increase the precipitation times. However, magnetic loops tend to become kink-instable when the twist gets too strong (e.g., Priest 1981) which puts natural limits on the amount of twisting.

Such trapping models predict a energy-dependent energy loss for the trapped particles. Since the measured spectral components originate from accelerated particles of different energies the precipitation times for the different spectral components should vary. For the trapping model calculated by Ramaty \& Mandzhavidze (1994) the following precipitation times are given for a pitch angle cosine of 0.5 and a loop length of $10^{7} \mathrm{~m}$. Protons with energies of several hundred $\mathrm{MeV}$ that are responsible for the pion decay signal should bounce 32000 times in such a trap, yielding a precipitation time of about $140 \mathrm{~min}$. Protons in the energy range of a few $10 \mathrm{MeV}$ that can excite nuclei causing the prompt nuclear line emission are expected to bounce only about 450 times and precipitate with a time constant of about $5.5 \mathrm{~min}$. The ratio of the precipitation times is $\tau_{\text {pions }} / \tau_{4-7}=25$.

For the short decay phase of the 11 June 1991 flare we have measured both precipitation times with COMPTEL and we find $\tau_{\text {pions }}=10.8 \pm 1.4 \mathrm{~min}$ and $\tau_{4-7}=12.1 \pm 1.1 \mathrm{~min}$. This gives a ratio of $\tau_{\text {pions }} / \tau_{4-7}=$ $0.89 \pm 0.15$. The measured ratio is far below the predicted 
value. During the long decay phase of the 11 June flare COMPTEL does not detect significant pion decay emission. However, Kanbach et al. (1993) deduced a decay time of $255 \mathrm{~min}$ from EGRET measurements. Together with our result of $\tau_{4-7}=220 \pm 160$ min we determined the ratio to be 1.15 , although with large statistical uncertainties. Both ratios are consistent with 1, implying that the precipitation rates of protons causing pionic decay radiation and nuclear line radiation are equal.

Comparison of pion decay (from several $100 \mathrm{MeV}$ protons) and 4-7 MeV radiation (from some $10 \mathrm{MeV}$ protons) is the most sensitive case. However, a trapping scenario would also predict to find (less pronounced) differences in the decline times of $2.223 \mathrm{MeV}$ (from about 20-100 MeV protons) and 4-7 MeV, as well as pion decay and $2.223 \mathrm{MeV}$ emission. Rank et al. (1996) compared the $2.223 \mathrm{MeV}$ signal measured by COMPTEL with the $>50 \mathrm{MeV}$ radiation measured by EGRET for the 11 June 1991 flare finding that the precipitation times do not differ.

Further, the trapped electrons should loose their energy much faster than the protons. The similarity between the $9.1 \mathrm{GHz}$ microwave signal and the $>50 \mathrm{MeV}$ GAMMA-1 measurements for the 15 June 1991 flare was noted by Akimov et al. (1994) as an argument against long-term trapping during this flare. For the 11 June 1991 flare it is clear from Table 2 that the bremsstrahlung signal originating from electron $>100 \mathrm{keV}$ declines in the same manner as all of the nucleonic components. Using the $2.223 \mathrm{MeV}$ data that allow the highest statistical significance we find a ratio of $\tau_{2.2} / \tau_{\text {brems }}=1.11 \pm 0.15$ for the short decay phase of the 11 June flare.

For the 11 June 1991 flare we have found for both decay phases of the extended emission, that the decline time ratio between each pair of the measured spectral components is consistent with a value of 1 , showing that both the proton spectrum and the $\mathrm{e} / \mathrm{p}$ ratio do not change significantly during the extended phase. Inspection of Table 2 shows also for the flares on 9 and 15 June 1991 that all ratios of the decline times between each pair of the measured spectral components is close to 1 . We conclude from this result that the measurements favour a continuous acceleration process.

A further argument against the trapping model comes from the emission characteristics of the pion decay radiation. If protons have been trapped from the impulsive phase and do not gain energy before the extended phase it is difficult to explain the absence of the $8-30 \mathrm{MeV}$ signal during the intermediate phase. The protons must have reached their energy during the impulsive phase and get trapped instantaneously and very efficiently so that they cannot produce a measurable pion decay signal before the extended phase starts. Further, the need for high plasma turbulence to accelerate the particles together with very low plasma turbulence in order to trap them efficiently makes it necessary that either the plasma conditions change dramatically, or that the particles are accelerated and trapped in different volumes.
Mandzhavidze et al. (1996) suggested an intermediate case of successive episodes of particle acceleration and subsequent trapping for moderately long times (termed "episodal acceleration"). On the time scales we can study we cannot rule this out.

The model by Ryan \& Lee (1991) explains the extended $\gamma$-ray emission by continuous acceleration of a particle population from the impulsive phase injected into turbulent large-scale loops. The particles scatter frequently with plasma waves and diffuse in space and momentum. For low turbulence levels the particles diffuse quickly and precipitate at the footpoints leading to an impulsive event. For a sufficiently high turbulence level the mean free path of the particles is much smaller than the loop dimensions. If the particles gain enough energy by second order Fermi acceleration to overcome energy losses the acceleration and slow precipitation can be maintained leading to extended $\gamma$-ray emission.

This acceleration model was initially suggested for the late phase of the 3 June 1982 flare and it can explain the 3 June 1982 data with a loop size of $10^{8} \mathrm{~m}$. However, when applied to the much more demanding requirements of the June 1991 flare (Ryan et al. 1993b; Bennett et al. 1994), loop sizes of $10^{9} \mathrm{~m}$ (3 solar radii) are necessary to keep the Alfvén speed at reasonable values and to avoid unrealistically high values of $\delta B / B$. Nevertheless, the model provides some characteristics which are interesting for the extended flare emission.

In this model, the particles continuously gain energy and will at some time reach the pion production threshold of about $400 \mathrm{MeV} /$ nucleon. This explains the onset of pion decay emission late in the flare without the need for a explicit two-step process. The acceleration process itself takes place continuously over a long time while the onset of the pionic emission only indicates that the energy of the accelerated particles has reached the threshold for pion production.

The $\gamma$-ray flux is expected to rise to a maximum and then decay exponentially with a decay constant independent of the spectrum of the seed population. The decay constant $\tau$ is given by

$\tau=\frac{l^{2}}{\pi^{2} \kappa}$

where $l$ is the size of the loop and $\kappa$ is the energyindependent spatial diffusion constant. If we assume that $\kappa$ is similar for the different loops, the different decay times that have been observed are explained by differences in the loop dimensions.

One interesting aspect of this acceleration process is that the decay time of the emission should be independent of the seed particle population. This feature of the model explains why the impulsive phases of the June 1991 flares differ, whereas the extended phases are similar.

One of the model assumptions is that turbulent conditions are present in magnetic loops after a flare occurred. However, the turbulence level in a loop is largely unobservable. Evidence for uniform turbulent reconnection along 
a $>5 \times 10^{8} \mathrm{~m}$ coronal loop has been reported by Priest et al. (1998). Only uniform heating along the loop can explain the measurements of pressure gradients. Such delocalized reconnection in a large flare loop could establish high turbulence levels or maintain the turbulent conditions found after a flare.

For the 15 June flare a different mechanism was proposed by Akimov et al. (1996). After a CME moves out of the corona, the disturbed magnetic field configuration restores its original shape due to magnetic reconnection. Hereby a current sheet forms high in the corona and can be the energy source for prolonged particle acceleration. It is noted by Akimov et al. (1996) that particle acceleration directly at the CME shock front is unlikely because it moves out too quickly and the particles could not return to the solar surface efficiently enough late in the event. In the case of the 9 and 11 June flare no evidence for a CME has been found.

This allows the interpretation that the presence of high energetic particles also during the extended phase on 15 June might be unrelated to the occurrence of the CME. On the other hand it might be speculated if the CME even disrupted the global magnetic configuration of AR-6659 so that this was the last in this remarkable series of flares. Unfortunately, AR-6659 rotated off the visible disk shortly after the 15 June event and no observations of structural changes at photospheric heights could be made.

One demanding observation for a model is the striking likeness of the long-term time profiles seen in $\gamma$-rays. Postflare loops systems have been observed for the flares on 9 and 11 June 1991 on disk from optical measurements (e.g., Sakurai et al. 1992). A very pronounced post-flare loop arcade was seen over the limb on 15 June 1991 (Akimov et al. 1996). It is also interesting that active region AR-6659 was a very stable group and could be observed already two solar rotations earlier as reported by Bumba et al. (1993). We speculate that the large scale loop systems remained intact during the two week observation period so that in the context of a continuous loop acceleration model the same loops could be populated more than one time. For a rising $\mathrm{CME}$ such a stationary scenario is not possible. It would be necessary instead that the acceleration region in the current sheet develops in a similar way during each flare and that the dimensions of the high-altitude loop systems are similar to produce similar decay times.

\section{Conclusions}

We present results of the $\gamma$-ray measurements of COMPTEL for the solar flares on 9, 11, and 15 June 1991. The most remarkable result is the observation of extended $\gamma$-ray emission for several hours after the flares. We studied the time profiles of different spectral components, and the development of the flare spectra. From the available observations we find the following characteristics for the three flares:

1. The extended phase differs from the impulsive phase in many respects, pointing to a different origin of the radiation. This was investigated in detail for the 11 June 1991 flare. The spectrum of the accelerated particle spectrum in the energy range of about $10-100 \mathrm{MeV}$ is harder during the extended phase, compared to the impulsive phase. Pions are not generated during the impulsive phase but a strong pion signal is found during the extended phase. The temporal structure during the extended phase is more smoothly varying. The hard X-ray emission can be described by a broken power law during the impulsive phase, and by a single power law with an additional superhot component during the extended phase. The power law index of the electrons is softer during the extended phase, and the e/p ratio is lower. To the extent these properties can be observed for the 9 June flare they yield similar results. One exception is the pion decay radiation, that is not detected with COMPTEL during the 9 June 1991 flare. No COMPTEL data are available during the impulsive phase of the 15 June 1991 flare.

2. The long-term time profiles of the extended phase for all three flares show a two-fold exponential decay curve in all energy bands that are measured with sufficient sensitivity to permit a fit. The exponential decay times are on the order of $10 \mathrm{~min}$ for the short and some $100 \mathrm{~min}$ for the long decay phase. For the 9 and 11 June flare the spectrum in the long decay phase is softer than in the short one. This effect was not observed for the 15 June 1991 flare, however. Also, the spectrum during the short decay phase slowly becomes softer for the 11 June flare. The same trend is found for the 9 June 1991 flare but is not significant. It was unobservable for the 15 June 1991 flare. In general, the spectrum shows few changes during the extended phase.

3. The comparison of all three flares reveals a striking similarity of the extended phase time profiles of the nuclear deexcitation lines and in particular the $2.223 \mathrm{MeV}$ neutron capture line. The impulsive phases show no such resemblance.

We summarize the characteristics of the extended phase that must be explained by acceleration and/or transport models: there must be a mechanism that accelerates energetic protons and electrons in about the same ratio over a time span of several hours. The highest energies we found are on the order of $10 \mathrm{MeV}$ for electrons (9 June 1991 flare), and several $100 \mathrm{MeV}$ for protons (11 and 15 June 1991 flares). The proton spectrum appears to be softer during the long decay phase than during the short decay phase, but both electron and proton spectra do not exhibit large or sudden changes. Each of the extended emission periods is preceeded by a large impulsive $\gamma$-ray line flare. Although the extended phase is rich in pion decay radiation this is not the case during the impulsive phase. The similarity of the flares demands a mechanism that is robust and does not require any exceptional conditions. Moreover, this process must be repeatable within a few days.

By comparing proposed models for explaining the extended emission with our observations we find that continuous acceleration models are better suited to reproduce 
the measurements. Although a trapping model can explain the long precipitation times of the June 1991 flares and the existence of multiple exponential decay constants, the similar decay times of radiation caused by protons of different energies, and also by protons and electrons is a strong argument against long-term trapping. Also, the fact that pion decay radiation is detected during the extended phase but not earlier runs counter to a trapping scenario.

The continuous acceleration of particles for a prolonged period has been proposed in different physical environments. The impulsive and extended phase are explained by different acceleration scenarios. The stochastic acceleration of particles in closed loops by high plasma turbulence occurs naturally, explaining the generation of pions late in the flare, the exponential shape of the decay curve, and the existence of different decay constants. The acceleration of particles in a current sheet that forms after a CME can also explain the long-term profiles, and the efficient acceleration of protons and ions. However, it is questionable whether this scenario can happen repetitively within a few days. Both scenarios employ the impulsive phase as a trigger and a source of seed particles. This may explain how the differing natures of the impulsive phases of the three flares can lead to similar extended phases.

Acknowledgements. The COMPTEL project is supported by the German government through DLR grant 50 QV 90968, by NASA under contract NAS5-26645, and by the Netherlands Organization for Scientific Research (NWO). The authors thank Richard Schwartz for his support with the BATSE data analysis, and appreciate the effort of all collegues in the COMPTEL collaboration, which also includes SRON-Utrecht, Utrecht, The Netherlands, and Astrophysics Division, ESTEC, Noordwijk, The Netherlands.

\section{References}

Akimov, V. V., Afanassyev, V. G., Belaousov, A. S., et al. 1991, Proc. 22nd ICRC, Dublin, 3, 73

Akimov, V. V., Leikov, N. G., Belov, A. V., et al. 1994, in High-energy solar phenomena, ed. J. M. Ryan, \& W. T. Vestrand, AIP Proc., 294, 106

Akimov, V. V., Ambrož, P., Belov, A. V., et al. 1996, Sol. Ph., 166,107

Bennett, E., Lee, M. A., \& Ryan, J. M. 1994, in High-energy solar phenomena, ed. J. M. Ryan, \& W. T. Vestrand, AIP Proc., 294, 118

Bumba, V., Klvana, M., Kalman, B., \& Gyori, L. 1993, A\&A, 276, 193

Chupp, E. L., Forrest, D. J., Ryan, J. M., et al. 1981, ApJ, 244, L171

Chupp, E. L., Debrunner, H., Flückiger, E., et al. 1987, ApJ, 318,913

Debrunner, H., Lockwood, J. A., Ryan, J. M., et al. 1993, Proc. 23rd ICRC, Calgary, 3, 115

Debrunner, H., Lockwood, J. A., Barat, C., et al. 1997, ApJ, 479, 997
Djantemirov, H. M., Galper, A. M., Khodarovich, A. M., et al. 1995, Proc. 24th ICRC, Rome, 4, 94

Dunphy, P. P., \& Chupp, E. L. 1994, in High-energy solar phenomena, ed. J. M. Ryan, \& W. T. Vestrand, AIP Proc., 294, 112

Dunphy, P. P., Chupp, E. L., Bertsch, D. L., et al. 1999, Sol. Ph., 187, 45

Fishman, G. J., Meegan, C. E., Wilson, R. B., et al. 1989, Proc. GRO Workshop, ed. W. N. Johnson, 2, 39

Hua, X.-M., \& Lingenfelter, R. E. 1987, ApJ, 323, 779

Kanbach, G., Bertsch, D. L., Fichtel, C. E., et al. 1993, A\&AS, 97, 349

Kocharov, G. E., Chuikin, E. I., Kovaltsov, G. A., et al. 1994, in High-energy solar phenomena, ed. J. M. Ryan, \& W. T. Vestrand, AIP Proc., 294, 45

Kocharov, L. E., Debrunner, H., Kovaltsov, G. A., et al. 1998, A\&A, 340, 257

Lau, Y.-T., \& Ramaty, R. 1994, in High-energy solar phenomena, ed. J. M. Ryan, \& W. T. Vestrand, AIP Proc., 294, 71

Lin, R. P., \& Schwartz, R. A. 1987, ApJ, 312, 462

Mandzhavidze, N., \& Ramaty, R. 1992, ApJ, 396, L111

Mandzhavidze, N., Ramaty, R., Akimov, V. V., et al. 1993, Proc. 23rd ICRC, Calgary, 3, 119

Mandzhavidze, N., Ramaty, R., Bertsch, D. L., \& Schneid, E. J., 1996, in High-energy solar physics, ed. R. Ramaty, N. Mandzhavidze, \& X.-M. Hua, AIP Proc., 374, 225

McConnell, M., Bennett, K., Forrest, D., et al. 1993, Adv. Sp. Res., 13(9), 248

Murphy, R. J., Dermer, C. D., \& Ramaty, R. 1987, ApJS, 63, 721

Murphy, R. J., Share, G. H., Letaw, J. R., \& Forrest, D. J. 1990, ApJ, 358, 298

Murphy, R. J., Share, G. H., Grove, J. E., et al. 1993, in Compton gamma-ray observatory, ed. M. Friedlander, N. Gehrels, \& D. J. Macomb, AIP Proc., 280, 619

Murphy, R. J., Share, G. H., Grove, J. E., et al. 1997, ApJ, 490,883

Nieminen, P. 1997, Ph.D. Thesis, Univ. Berne

Prince, T. A., Forrest, D. J., Chupp, E. L., Kanbach, G., \& Share, G. H. 1983, Proc. 18th ICRC, Bangalore, 4, 79

Priest, E. R. 1981, Solar flare magnetohydrodynamics, ed. E. R. Priest (Gordon and Breach)

Priest, E. R., Foley, C. R., Heyvearts, J., et al. 1998, Nature, 393, 545

Ramaty, R., Mandzhavidze, N., Kozlovski, B., et al. 1993, Adv. Sp. Res. 13, 275

Ramaty, R., \& Mandzhavidze, N. 1994, in High-energy solar phenomena, ed. J. M. Ryan, \& W. T. Vestrand, AIP Proc., 294,26

Ramaty, R., \& Mandzhavidze, N. 1996, in High-energy solar physics, ed. R. Ramaty, N. Mandzhavidze, \& X.-M. Hua, AIP Proc., 374, 533

Rank, G., Diehl, R., Lichti, G. G., et al. 1993, in Compton gamma-ray observatory, ed. M. Friedlander, N. Gehrels, \& D. J. Macomb, AIP Proc., 280, 661

Rank, G., Diehl, R., Lichti, G. G., et al. 1994, in High-energy solar phenomena, ed. J. M. Ryan, \& W. T. Vestrand, AIP Proc., 294, 100

Rank, G., Bennett, K., Bloemen, H., et al. 1996, in High-energy solar physics, ed. R. Ramaty, N. Mandzhavidze, \& X.-M. Hua, AIP Proc., 374, 219 
Rank, G. 1996, Ph.D. Thesis, Techn. Univ. Munich

Rank, G., Debrunner, H., Lockwood, J., et al. 1997, Proc. 25th ICRC, 1,5

Ryan, J. M., \& Lee, M. A. 1991, ApJ, 368, 316

Ryan, J. M., Forrest, D. J., Lockwood, J., et al. 1993a, in Compton gamma-ray observatory, ed. M. Friedlander, N. Gehrels, \& D. J. Macomb, AIP Proc., 280, 631

Ryan, J. M., Bennett, E., \& Lee, M. A. 1993b, Adv. Sp. Sc., 13,255

Ryan, J. M., Forrest, D. J., Lockwood, J., et al. 1994, in High-energy solar phenomena, ed. J. M. Ryan, \& W. T. Vestrand, AIP Proc., 294, 89

Sakurai, T., Ichimoto, K., Hiei, E., et al. 1992, PASJ, 44, L7
Schneid, E. J., Brazier, K. T. S., Kanbach, G., et al. 1994, in High-energy solar phenomena, ed. J. M. Ryan, \& W. T. Vestrand, AIP Proc., 294, 94

Schönfelder, V., Bennett, K., Bloemen, H., et al. 1993, ApJS, 86,657

Stacy, J. G., Kippen, R. M., Kappadath, S. C., et al. 1996, A\&AS, 120, 691

Strong, A. W., Bennett, K., Bloemen, H., et al. 1993, A\&AS, 97, 133

Trottet, G., Vilmer, N., Barat, C., et al. 1993, A\&AS, 97, 337

Trottet, G. 1994, in High-energy solar phenomena, ed. J. M. Ryan, \& W. T. Vestrand, AIP Proc., 294, 3

van Dijk, R. 1996, Ph.D. Thesis, Univ. Leiden 